\title{
Approximation of the effective conductivity of ergodic media by periodization.
}

\author{
Houman Owhadi*
}

July 22, 2013

\begin{abstract}
This paper is concerned with the approximation of the effective conductivity $\sigma(A, \mu)$ associated to an elliptic operator $\nabla_{x} A(x, \eta) \nabla_{x}$ where for $x \in \mathbb{R}^{d}, d \geq 1, A(x, \eta)$ is a bounded elliptic random symmetric $d \times d$ matrix and $\eta$ takes value in an ergodic probability space $(X, \mu)$. Writing $A^{N}(x, \eta)$ the periodization of $A(x, \eta)$ on the torus $T_{N}^{d}$ of dimension $d$ and side $N$ we prove that for $\mu$-almost all $\eta$

$$
\lim _{N \rightarrow+\infty} \sigma\left(A^{N}, \eta\right)=\sigma(A, \mu)
$$

We extend this result to non-symmetric operators $\nabla_{x}(a+E(x, \eta)) \nabla_{x}$ corresponding to diffusions in ergodic divergence free flows ( $a$ is $d \times d$ elliptic symmetric matrix and $E(x, \eta)$ an ergodic skew-symmetric matrix); and to discrete operators corresponding to random walks on $\mathbb{Z}^{d}$ with ergodic jump rates.

The core of our result is to show that the ergodic Weyl decomposition associated to $\mathrm{E}^{2}(X, \mu)$ can almost surely be approximated by periodic Weyl decompositions with increasing periods, implying that semi-continuous variational formulae associated to $\mathrm{E}^{2}(X, \mu)$ can almost surely be approximated by variational formulae minimizing on periodic potential and solenoidal functions.
\end{abstract}

\section{Contents}

1 Introduction

AMS 1991 Subject Classification. Primary 74Q20, 37A15; secondary 37A25

Key words and phrases. Effective conductivity, periodization of ergodic media, Weyl decomposition

*LATP, UMR CNRS 6632, CMI, Université de Provence, owhadi@cmi.univ-mrs.fr 
2 General set up 4

2.1 The ergodic space . . . . . . . . . . . . . . 4

2.1 .1 Continuous case . . . . . . . . . . . . . . . . 4

2.1 .2 Discrete case . . . . . . . . . . . . . . . . . . 5

2.2 Wevl Decomposition . . . . . . . . . . . . . . 5

2.2.1 Continuous case . . . . . . . . . . . . . 5

2.2 .2 Discrete case . . . . . . . . . . . . . 6

$\begin{array}{lll}3 & \text { Main result } & 6\end{array}$

3.1 Almost sure continuitv of Wevl's decomposition . . . . . . . . 6

3.1 .1 Proof of theorem $3.1 \ldots \ldots . \ldots 9$

3.2 Periodic approximation of variational functionals on the ergodic spaces 11

4 Application 14

4.1 Svmmetric continuous Operator . . . . . . . . . . . . . . . 14

4.1.1 Homogenization in the ergodic medium . . . . . . . 14

4.1.2 Periodization of the ergodic medium . . . . . . . . 14

4.1.3 The main theorem . . . . . . . . . . . 15

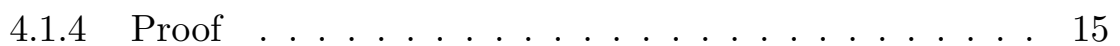

4.2 Non svmmetric continuous Operator, diffusion in divergence free flow 16

4.2.1 Homogenization in the ergodic medium . . . . . . 16

4.2.2 Periodization of the ergodic medium . . . . . . . . 17

4.2 .3 The main theorem . . . . . . . . . . . 18

4.2.4 Core of the proof: Variational formulations and theorem 4.18

4.3 Discrete Operator . . . . . . . . . . . . . . . . . 20

4.3.1 The ergodic homogenization problem . . . . . . . . . 20

4.3.2 Periodization of the ergodic medium . . . . . . . 20

4.3.3 The main theorem . . . . . . . . . . . . . . . 21

4.3 .4 Proof . . . . . . . . . . . . . . . . 21

5 Proofs 22

5.1 Main results . . . . . . . . . . . . . . . . . . 22

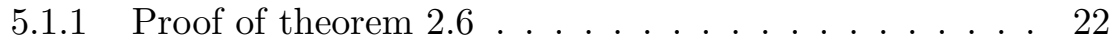

5.1.2 Proof of equation (22) of lemma 3.3 in the discrete case 23

5.1.3 Proof of equation (21) of lemma 3.3 in the discrete case 26

5.1.4 Proof of equation (21) of lemma 3.3 in the continuous case 27

5.1.5 Proof of equation (22) of lemma 3.3 in the continuous case 32

5.2 Applications . . . . . . . . . . . . . . . 32

5.2.1 Proof of the variational formula 851] . . . . . . . 32

5.2.2 Proof of the variational formulas (86) and (87) . . . 34

5.2 .3 Proof of lemma $4.7 \ldots \ldots \ldots$ 


\section{Introduction}

Homogenization theory has been developed to find the asymptotic behavior of operators associated to an heterogeneous ergodic medium when the microscopic scale associated to the heterogeneities tends towards 0 in front of the macroscopic scale of the observation. The mathematical formulation of this theory BLP78 has been first developed in the simpler case of elliptic and parabolic periodic operators. The first rigorous results on elliptic and stationary parabolic ergodic operators were obtained by S. Kozlov Koz80, Koz85, G. Papanicolaou and S. Varadhan PV79] in the late seventies. Next C. Kipnis and S.R.S. Varadhan KV86 followed by MFGW89 and OS95 introduced powerful central limit theorems allowing the extension of homogenization theory to a wide range of ergodic operators.

Thus two main categories of problems have been addressed by homogenization theory: the asymptotic behavior of periodic operators and the asymptotic behavior of ergodic operators. The question of the existence of a natural and continuous link between those two categories of applications has naturally arisen. Indeed for large deviations DGI00 and equilibrium fluctuations GOS01 of $\nabla \phi$ interface models it has been observed that the regularity of the effective conductivity associated to the infinite dimensional ergodic system under its finite dimensional periodic approximations hides an hard core difficulty in extending the mathematical description of relaxation towards equilibrium of periodic environments to ergodic ones.

Recently this regularity property has been proven for the self-diffusion coefficient for the exclusion process [LOV01]. The case of the effective diffusivity of a symmetric random walk on $\mathbb{Z}^{d}$, under the condition that its jump rates are i.i.d. has been addressed in CI01, which also put into evidence an exponential rate of convergence of effective diffusivities of the finite volume approximations of the ergodic medium.

It is important to note that the periodic approximation of the effective conductivity of a continuous ergodic operator can be obtained from almost sure $G$-convergence properties associated to the homogenization of that operator; this is the so called "principle of periodic localization" (equation 5.15, page 155 of JKO91, Pia02]).

It is well known that these effective conductivities associated to periodic and ergodic operators are given by variational formulae in the geometrical framework of Weyl decomposition. Weyl decomposition plays a central role in homogenization in periodic or ergodic media but is independent of them. Thus it is natural to seek for a geometrical property inherent to Weyl decomposition allowing the almost sure approximation of ergodic effective conductivities by periodization. The purpose of our paper is to show that such property does exist: the Weyl decomposition is gifted with almost sure strong stability properties (theorem 3.1, proposition 3.2) which can be used 
to establish a natural and continuous link between homogenization in periodic and ergodic media. We refer to theorem 4.1 for a continuous operator, theorem 4.2 for a non symmetric operator and 4.6 for discrete operator (one can also consider a larger class of homogenization problems such as those associated to the lemma 3.1 of [Nor97]). This property inherent to Weyl decomposition can also be used to obtain almost sure periodic approximation results for a wider class of variational formulations on an ergodic space than those associated to an effective conductivity, this is the object of theorem 3.8 .

\section{General set up}

\subsection{The ergodic space}

\subsubsection{Continuous case}

Let $(X, \mathcal{G}, \mu)$ be a probability space with $\eta \in X$ labeling the particular realization of the quenched medium. We assume that on $(X, \mathcal{G}, \mu)$ acts ergodically a group of measure preserving transformations $G=\left\{\tau_{x}: x \in\right.$ $\mathbb{R}^{d}$ \}, i.e. that the following are satisfied:

Condition 2.1. $\forall x \in \mathbb{R}^{d}, \tau_{x}$ preserves the measure, namely, $\forall A \in \mathcal{G}$, $\mu\left(\tau_{x} A\right)=\mu(A)$;

Condition 2.2. The action of $G$ is ergodic, namely, if $A=\tau_{x} A \forall x \in \mathbb{R}^{d}$, then $\mu(A)=0$ or $\mu(A)=1$.

Let $L^{2}(\mu)$ be the Hilbert space of square integrable functions on $\mathrm{X}$ with the usual scalar product

$$
\int_{X} f(\eta) g(\eta) d \mu(\eta)
$$

Let $f \in L^{2}(\mu)$, for almost every $\eta$ we define

$$
\left(T_{x} f\right)(\eta)=f\left(\tau_{-x} \eta\right)
$$

We assume furthermore that

Condition 2.3. For any measurable function $f(\eta)$ on $X$, the function $T_{x} f(\eta)$ defined on the Cartesian product $X \times \mathbb{R}^{d}$ is also measurable (where $\mathbb{R}^{d}$ is endowed with the Lebesgue measure).

It follows that that $T_{x}$ form a strongly continuous unitary group on $L^{2}(\mu)$ (see [JKO91] chapter 7).

For $f \in L^{1}(\mu)$ we write.

$$
<f>\equiv \int_{X} f(\eta) \mu(d \eta)
$$




\subsubsection{Discrete case}

We shall distinguish through this paper two cases of ergodic spaces. The one mentioned above associated to a continuous of measure preserving transformations and the one mentioned here associated to a discrete measure preserving transformations. We shall keep the same notation used above for the continuous case. $(X, \mathcal{G}, \mu)$ will remain our ergodic probability space with $\eta \in X$ labeling the particular realization of the quenched medium but we replace the group of measure preserving transformations acting ergodically on $(X, \mathcal{G}, \mu)$ by $G=\left\{\tau_{x}: x \in \mathbb{Z}^{d}\right\}$. We will replace the conditions 2.1 and 2.2 by

Condition 2.4. $\forall x \in \mathbb{Z}^{d}$, $\tau_{x}$ preserves the measure, namely, $\forall A \in \mathcal{G}$, $\mu\left(\tau_{x} A\right)=\mu(A)$;

Condition 2.5. The action of $G$ is ergodic, namely, if $A=\tau_{x} A \forall x \in \mathbb{Z}^{d}$, then $\mu(A)=0$ or $\mu(A)=1$.

\subsection{Weyl Decomposition}

\subsubsection{Continuous case}

A vector field $f=\left(f_{1}, \ldots, f_{d}\right), f_{i} \in L_{l o c}^{2}\left(\mathbb{R}^{d}\right), i=1, \ldots, d$ is called vortexfree in $\mathbb{R}^{d}$ if

$$
\int_{\mathbb{R}^{d}}\left(f_{i} \partial_{j} \phi-f_{j} \partial_{i} \phi\right) d x=0 \quad \forall \phi \in C_{0}^{\infty}\left(\mathbb{R}^{d}\right)
$$

It is well known that any vortex-free vector possesses a potential function, i.e., admits the representation $f=\nabla u, u \in H_{l o c}^{1}\left(\mathbb{R}^{d}\right)$. Therefore the potentiality of a vector field $f$ is equivalent to the property (44). A vector field $f$ is said to be solenoidal in $\mathbb{R}^{d}$ if

$$
\int_{\mathbb{R}^{d}} f_{i} \partial_{i} \phi(x) d x=0, \quad \forall \phi \in C_{0}^{\infty}\left(\mathbb{R}^{d}\right)
$$

Now let us consider vector fields on $X$. A vector field $f \in\left(L^{2}(\mu)\right)^{d}=$ $\mathrm{E}^{2}(X, \mu)$ will be called potential (resp., solenoidal), if almost all its realizations $T_{x} f(\eta)$ are potential (resp., solenoidal) in $\mathbb{R}^{d}$. The spaces of potential and solenoidal vector fields denoted by $\mathrm{E}_{p o t}^{2}(X, \mu)$ and $\mathrm{E}_{\text {sol }}^{2}(X, \mu)$, form closed sets in $\mathrm{E}^{2}(X, \mu)$.

Set

$$
\begin{aligned}
& F_{\text {pot }}^{2}=\left\{f \in \mathrm{E}_{\text {pot }}^{2}(X, \mu),<f>=0\right\} \\
& F_{\text {sol }}^{2}=\left\{f \in \mathrm{E}_{\text {sol }}^{2}(X, \mu),<f>=0\right\}
\end{aligned}
$$

By Weyl's decomposition (see the lemma 7.3 of [JKO91]) the following orthogonal decomposition are valid

$$
\mathrm{E}^{2}(X, \mu)=F_{\text {pot }}^{2} \oplus F_{\text {sol }}^{2} \oplus \mathbb{R}^{d}=F_{\text {pot }}^{2}+\mathrm{E}_{\text {sol }}^{2}(X, \mu)
$$




\subsubsection{Discrete case}

For any $f: X \rightarrow \mathbb{R}$ and $i \in\{1, \ldots, d\}$ we write

$$
D_{i} f(\eta)=f\left(\tau_{-e_{i}} \eta\right)-f(\eta) \quad D_{i}^{*} f(\eta)=D_{i} f\left(\tau_{e_{i}} \eta\right)
$$

Write

$$
\mathrm{L}^{2}(X, \mu):=\left\{\left(f_{i}\right)_{1 \leq i \leq d}: f_{i} \in L^{2}(\mu)\right\}
$$

and $F_{\text {pot }}^{2}$ the completion of $\left\{\left(D_{i} f(\eta)\right)_{1 \leq i \leq d}: f \in L^{2}(\mu)\right\}$ in $\mathrm{E}^{2}(X, \mu)$ with respect to the standard $L^{2}$ norm $\left(\|f\|^{2}=\sum_{i=1}^{d}\left\langle f_{i}^{2}\right\rangle\right)$.

Write $\mathcal{S}(X, \mu)$ the set of skew-symmetric matrices $H$ such that $H_{i, j} \in L^{2}(\mu)$ and define $\mathcal{D}$ iv $H$ as the vector $(\mathcal{D} \text { iv } H)_{i}:=\sum_{j=1}^{d} D_{j} H_{i j}$. We write $F_{\text {sol }}^{2}$ the completion of $\{\mathcal{D}$ iv $H: H \in \mathcal{S}(X, \mu)\}$ in $\mathrm{L}^{2}(X, \mu)$ with respect to the standard $L^{2}$ norm.

We will prove the subsection 5.1.1 the following theorem corresponding to the Weyl decomposition.

Theorem 2.6. One has

$$
E^{2}(X, \mu)=F_{\text {pot }}^{2} \oplus F_{\text {sol }}^{2} \oplus \mathbb{R}^{d}
$$

\section{Main result}

\subsection{Almost sure continuity of Weyl's decomposi- tion}

The results and notations given in this section are valid in the continuous case as in the discrete case. Write $T_{N}^{d}$ the torus of side $N$ and dimension $d\left(T_{N}^{d}:=\mathbb{R}^{d} /\left(N \mathbb{Z}^{d}\right)\right.$ in the continuous case and $T_{N}^{d}:=\mathbb{Z}^{d} /\left(N \mathbb{Z}^{d}\right)$ in the discrete). Write $\mathrm{E}^{2}\left(T_{N}^{d}\right)$ the space of square integrable vectors fields on $T_{N}^{d}$ and gift it with the norm $\|f\|_{\mathrm{E}^{2}\left(T_{N}^{d}\right)}^{2}$ (written below) to obtain an Hilbert space. We define

$$
\|f\|_{\mathrm{E}^{2}\left(T_{N}^{d}\right)}^{2}:=\sum_{i=1}^{d} N^{-d} \int_{T_{N}^{d}}\left(f_{i}(x)\right)^{2} d x
$$

in the continuous case and

$$
\|f\|_{\mathrm{E}^{2}\left(T_{N}^{d}\right)}^{2}:=\sum_{i=1}^{d} N^{-d} \sum_{x \in T_{N}^{d}}\left(f_{i}(x)\right)^{2}
$$

in the discrete case. In the continuous case, write $F_{\text {pot }}^{2}\left(T_{N}^{d}\right)\left(F_{\text {sol }}^{2}\left(T_{N}^{d}\right)\right)$ the completion of the space of smooth $T_{N}^{d}$-periodic potential (solenoidal) forms 
(with 0-Lebesgue mean value) in $\mathrm{E}^{2}\left(T_{N}^{d}\right)$ with respect to that norm. In the discrete case we shall use the following definitions

$$
F_{p o t}^{2}\left(T_{N}^{d}\right):=\left\{\nabla f: f \in L^{2}\left(T_{N}^{d}\right)\right\}
$$

Where $L^{2}\left(T_{N}^{d}\right)$ is the space of square integrable functions on $T_{N}^{d}$ and $\nabla$ is the discrete gradient on $\mathbb{Z}^{d},(\nabla f)_{i}:=\left(\nabla_{i} f\right)=f\left(x+e_{i}\right)-f(x)$. For $N$, let us write $\mathcal{S}\left(T_{N}^{d}\right)$ the set of skew-symmetric matrices with coefficients in $L^{2}\left(T_{N}^{d}\right)$ and for $H \in \mathcal{S}\left(T_{N}^{d}\right), \operatorname{div} H$ is the vector field defined by $(\operatorname{div} H)_{i}=$ $\sum_{j=1}^{d} \nabla_{j} H_{i, j}$.

$$
F_{\text {sol }}^{2}\left(T_{N}^{d}\right):=\left\{\operatorname{div} H: H \in \mathcal{S}\left(T_{N}^{d}\right)\right\}
$$

As in the ergodic case it is easy (see [JKO91]) to obtain the following Weyl decomposition for the periodic case:

$$
\mathrm{Ł}^{2}\left(T_{N}^{d}\right)=F_{\text {pot }}^{2}\left(T_{N}^{d}\right) \oplus F_{\text {sol }}^{2}\left(T_{N}^{d}\right) \oplus \mathbb{R}^{d}
$$

For any $\xi \in \mathrm{E}^{2}(X, \mu)$ we write $\Pi_{N} \xi \in \mathrm{E}^{2}\left(T_{N}^{d}\right)$ its periodization on the torus $T_{N}^{d}$ : for $x=p N+y$ with $p \in \mathbb{Z}^{d}$ and $y \in\left[0, N\left(^{d}\left(y \in \mathbb{Z} \cap\left[0, N\left(^{d}\right.\right.\right.\right.\right.$ in the discrete case)

$$
\Pi_{N} \xi(x, \eta):=T_{y} f(\eta)
$$

Let us define $\lim _{N \rightarrow \infty} F_{\text {pot }}^{2}\left(T_{N}^{d}\right)$ as the subset of $\xi \in \mathrm{E}^{2}(X, \mu)$ such that for $\mu$-almost all $\eta \in X$ there exists a sequence $\nu_{\text {pot }}^{N} \in F_{\text {pot }}^{2}\left(T_{N}^{d}\right)$ such that

$$
\lim _{N \rightarrow \infty}\left\|\Pi_{N} \xi(\eta)-\nu_{p o t}^{N}\right\|_{\complement^{2}\left(T_{N}^{d}\right)}=0
$$

Similarly we define $\lim _{N \rightarrow \infty} F_{\text {sol }}^{2}\left(T_{N}^{d}\right)$ as the subset of $\xi \in \mathrm{E}^{2}(X, \mu)$ such that for $\mu$-almost all $\eta \in X$ there exists a sequence $\nu_{\text {sol }}^{N} \in F_{\text {sol }}^{2}\left(T_{N}^{d}\right)$ such that

$$
\lim _{N \rightarrow \infty}\left\|\Pi_{N} \xi(\eta)-\nu_{s o l}^{N}\right\|_{\complement^{2}\left(T_{N}^{d}\right)}=0
$$

The following theorem (valid for both continuous and discrete cases) is the central point linking homogenization in ergodic media to homogenization in periodic media.

\section{Theorem 3.1.}

$$
\lim _{N \rightarrow \infty} F_{p o t}^{2}\left(T_{N}^{d}\right)=F_{p o t}^{2}
$$

and

$$
\lim _{N \rightarrow \infty} F_{\text {sol }}^{2}\left(T_{N}^{d}\right)=F_{\text {sol }}^{2}
$$

Where the limits in $N$ are taken along $\mathbb{R}^{+}$in the continuous case and along $\mathbb{N}$ in the discrete. 
For any $\xi \in \mathrm{E}^{2}(X, \mu)$ we write $\xi_{\text {pot }}$ and $\xi_{\text {sol }}$ its components on $F_{p o t}^{2}$ and $F_{\text {sol }}^{2}$. For any $\nu \in \mathrm{E}^{2}\left(T_{N}^{d}\right)$ we write $\nu_{\text {pot }}$ and $\nu_{\text {sol }}$ its components on $F_{\text {pot }}^{2}\left(T_{N}^{d}\right)$ and $F_{\text {sol }}^{2}\left(T_{N}^{d}\right)$. The theorem 3.1 is based on the following proposition

Proposition 3.2. For any $\xi \in E^{2}(X, \mu)$, for $\mu$-almost all $\eta \in X$

$$
\lim _{N \rightarrow \infty}\left\|\Pi_{N}\left(\xi_{\text {pot }}(\eta)\right)-\left(\Pi_{N} \xi(\eta)\right)_{p o t}\right\|_{E^{2}\left(T_{N}^{d}\right)}=0
$$

and

$$
\lim _{N \rightarrow \infty}\left\|\Pi_{N}\left(\xi_{\text {sol }}(\eta)\right)-\left(\Pi_{N} \xi(\eta)\right)_{s o l}\right\|_{E^{2}\left(T_{N}^{d}\right)}=0
$$

Where the limits in $N$ are taken along $\mathbb{R}^{+}$in the continuous case and along $\mathbb{N}$ in the discrete.

In order to prove 20 observe that it is sufficient to prove the following lemma

Lemma 3.3. for any $\xi \in E^{2}(X, \mu)$, for $\mu$-almost all $\eta \in X$

$$
\lim _{N \rightarrow \infty}\left\|\Pi_{N}\left(\xi_{\text {pot }}(\eta)\right)-\left(\Pi_{N} \xi_{\text {pot }}(\eta)\right)_{\text {pot }}\right\|_{E^{2}\left(T_{N}^{d}\right)}=0
$$

and

$$
\lim _{N \rightarrow \infty}\left\|\Pi_{N}\left(\xi_{\text {sol }}(\eta)\right)-\left(\Pi_{N} \xi_{\text {sol }}(\eta)\right)_{\text {sol }}\right\|_{E^{2}\left(T_{N}^{d}\right)}=0
$$

Where the limits in $N$ are taken along $\mathbb{R}^{+}$in the continuous case and along $\mathbb{N}$ in the discrete.

There exists two strategies two prove lemma 3.3. For the equation (21) for instance, the second strategy consists in taking the primitive of $\xi_{p o t}$, then approximating that primitive by a $T_{N}^{d}$-periodic function whose gradient is used to approximate $\Pi_{N}\left(\xi_{\text {pot }}(\eta)\right)$. This second strategy is the harder one because the primitive of $\xi_{\text {pot }}$ is in general not an element of $\mathrm{E}^{2}(X, \mu)$. The first strategy consists in finding a function $u$ of $L^{2}(\mu)$ such that its gradient is an approximation of $\xi_{\text {pot }}$ then proving (21) for $\nabla u$. Our proof of the Weyl decomposition (theorem [2.6) and the definition of $F_{p o t}^{2}$ allows us to use the simpler first strategy in the discrete case. In the continuous case we have used the second strategy (which can also be used for the discrete case) but one can also adapt the second strategy to the continuous case (it is important to note that first, one has to prove that there exists a subset of $\mathrm{E}^{2}(X, \mu)$ such that the gradients of its elements are dense in $\left.F_{\text {pot }}^{2}\right)$. The proof of lemma 3.3 will be given in subsection [5.1. We will first prove in subsection 5.1.2 equation (22) in the discrete case using the first strategy, the proof of equation (21) in the discrete case being similar we will just give the idea of the proof in subsection 5.1.3. Then in subsection 5.1.4 we will prove equation (21) in the continuous case using the second strategy (the proof of equation (22) being similar we will just give its idea in subsection 5.1.4.1). 
Remark 3.4. Let us note that the proof of lemma 3.3 with the second strategy is constructive and Birkhoff ergodic theorem is applied only to $\xi_{p o t}$ and not to a sequence of its approximations. Thus the second strategy associate the rate at which Birkhoff ergodic theorem holds for $\xi_{\text {pot }}$ to the rate at which the limits in lemma 3.3 hold. More precisely, writing for $M \in \mathbb{N}$, $I(M)=\left\{\left(i_{1}, \ldots, i_{d}\right) \in\{1, \ldots, M\}^{d} ; \min _{j} \min \left(i_{j}-1, M-i_{j}\right)=0\right\}$ and $\mathcal{J}(M)$ the set of cubes $B_{i}$ indexed by $i \in I(M)$ and

$$
B_{i}=\left\{x \in[0,1]^{d}: \max _{j}\left|x_{j}-\left(i_{j}-0.5\right) / M\right| \leq 1 /(2 M)\right\}
$$

one can define

$$
\begin{gathered}
f\left(N, \xi_{\text {pot }}, \eta\right)=\sup \left\{M \in \mathbb{N}: \sup _{B_{i} \in \mathcal{J}\left(M^{2}\right)} M\left(\operatorname{Vol}\left(B_{i}\right)\right)^{-1}\left|\int_{B_{i}} \xi_{\text {pot }}(N x, \eta) d x\right| \leq 1\right. \text { and } \\
\left.\sup _{B_{i} \in \mathcal{J}(M)}\left(\operatorname{Vol}\left(B_{i}\right)\right)^{-1} \int_{B_{i}}\left|\xi_{\text {pot }}(N x, \eta)\right|^{2} d x \leq 2\left\langle\xi_{\text {pot }}^{2}\right\rangle\right\}
\end{gathered}
$$

It is then easy to check by Birkhoff ergodic theorem 3.5 that a.s. $f\left(N, \xi_{\text {pot }}, \eta\right) \rightarrow$ $\infty$ as $N \rightarrow \infty$ and by taking $M=f\left(N, \xi_{p o t}, \eta\right)$ and $P=M$ in subsection 5.1.4 one obtains that for all $\xi_{\text {pot }} \in F_{\text {pot }}^{2}$ and $N>0\left(N \in \mathbb{N}^{*}\right.$ in the discrete case)

$$
\left\|\Pi_{N}\left(\xi_{p o t}(\eta)\right)-\left(\Pi_{N} \xi_{p o t}(\eta)\right)_{p o t}\right\|_{\mathrm{E}^{2}\left(T_{N}^{d}\right)} \leq C_{d}\left\langle\xi_{p o t}^{2}\right\rangle^{\frac{1}{2}}\left(f\left(N, \xi_{p o t}, \eta\right)\right)^{-\frac{1}{2}}
$$

The same rate of convergence can be obtained with the second strategy in the discrete case for both equations (19) and (20). Then following the proof of our applications one can relate the rate at which the effective conductivity can be approximated by its periodizations to the function $f\left(N, \xi_{p o t}, \eta\right)$.

\subsubsection{Proof of theorem 3.1}

We will now give the proof of theorem 3.1 based on proposition 3.2. We will first give the proof in the continuous case. First, let us remind the standard ergodic theorem that we will use.

Let $f(x) \in L_{\text {loc }}^{1}\left(\mathbb{R}^{d}\right)$. A number $M\{f\}$ is called the mean value of $f$ if

$$
\lim _{\epsilon \rightarrow 0} \int_{K} f\left(\epsilon^{-1} x\right) d x=|K| M\{f\}
$$

for any Lebesgue measurable bounded set $K \subset \mathbb{R}^{d}$ (here $|K|$ stands for the Lebesgue measure of $K)$. Let $K_{t}=\left\{x \in \mathbb{R}^{d}, t^{-1} x \in K\right\}$ denote the homothetic dilatation, with ratio $t>0$, of the set $K$. Then (26) can be written in a more habitual form:

$$
\lim _{t \rightarrow \infty} \frac{1}{t^{d}|K|} \int_{K_{t}} f(x) d x=M\{f\}
$$


The following theorem is the theorem 10 of the chapter VIII.7.10 of DS67] (see also the theorem 7.2 of [JKO91] ).

Theorem 3.5. Let $f \in L^{p}(\mu)$ with $1 \leq p<\infty$. Then for almost all $\eta \in X$ the realization $T_{x} f(\eta)$ posses a mean value in the sense of (27). Moreover, the mean value $M\left\{T_{x} f(\eta)\right\}$, considered as a function of $\eta \in X$ is invariant, and for almost all $\eta \in X$

$$
<f>\equiv \int_{X} f(\eta) \mu(d \eta)=M\left\{T_{x} f(\eta)\right\}
$$

If $p>1$, then the limit in (27) also exists in the norm of $L^{p}$ and the functions are for $t>0$, all dominated by a function in $L^{p}$.

Now let us observe that from equation 19 one easily obtains that

$$
F_{\text {pot }}^{2} \subset \lim _{N \rightarrow \infty} F_{\text {pot }}^{2}\left(T_{N}^{d}\right)
$$

similarly from equation 20] one easily obtains that

$$
F_{\text {sol }}^{2} \subset \lim _{N \rightarrow \infty} F_{\text {sol }}^{2}\left(T_{N}^{d}\right)
$$

Now let $\xi \in \lim _{N \rightarrow \infty} F_{\text {pot }}^{2}\left(T_{N}^{d}\right)$ and $\nu \in \mathrm{E}_{\text {sol }}^{2}\left(T_{N}^{d}\right)$. Using the ergodic theorem 3.5. one has $\mu$-a.s.

$$
\lim _{N \rightarrow \infty} N^{-d} \int_{T_{N}^{d}} \Pi_{N} \xi(x, \eta) \cdot \Pi_{N} \nu(x, \eta) d x=\langle\xi . \nu\rangle
$$

Using

$$
\begin{aligned}
\int_{T_{N}^{d}} \Pi_{N} \xi(x, \eta) \cdot \Pi_{N} \nu(x, \eta) d x & =\int_{T_{N}^{d}}\left(\Pi_{N} \xi(x, \eta)\right)_{p o t} \cdot\left(\Pi_{N} \nu(x, \eta)\right)_{p o t} d x \\
& +\int_{T_{N}^{d}}\left(\Pi_{N} \xi(\eta)-\left(\Pi_{N} \xi(\eta)\right)_{p o t}\right) \cdot \Pi_{N} \nu(x, \eta) d x
\end{aligned}
$$

it follows that

$$
\begin{aligned}
& \mid N^{-d} \int_{T_{N}^{d}} \Pi_{N} \xi(x, \eta) . \Pi_{N} \nu(x, \eta) d x \mid \leq\left\|\left(\Pi_{N} \xi(\eta)\right)_{p o t}\right\|_{\mathrm{E}^{2}\left(T_{N}^{d}\right)}\left\|\left(\Pi_{N} \nu(\eta)\right)_{p o t}\right\|_{\mathrm{E}^{2}\left(T_{N}^{d}\right)} \\
&+\left\|\Pi_{N}(\xi(\eta))-\left(\Pi_{N} \xi(\eta)\right)_{p o t}\right\|_{\mathrm{E}^{2}\left(T_{N}^{d}\right)}\left\|\left(\Pi_{N} \nu(\eta)\right)\right\|_{\mathrm{E}^{2}\left(T_{N}^{d}\right)}
\end{aligned}
$$

Using the ergodic theorem 3.5] and proposition 3.2. one easily obtains that $\mu$-a.s.

$$
\lim _{N \rightarrow \infty} N^{-d} \int_{T_{N}^{d}} \Pi_{N} \xi(x, \eta) \cdot \Pi_{N} \nu(x, \eta) d x=0
$$


which proves that $\langle\xi . \nu\rangle=0$. Thus $\xi \perp \mathrm{E}_{\text {sol }}^{2}(X, \mu)$, which implies from Weyl decomposition that $\xi \in F_{\text {pot }}^{2}(X, \mu)$. Thus we have proven that

$$
\lim _{N \rightarrow \infty} F_{p o t}^{2}\left(T_{N}^{d}\right) \subset F_{p o t}^{2}
$$

Similarly one proves that

$$
\lim _{N \rightarrow \infty} F_{\text {sol }}^{2}\left(T_{N}^{d}\right) \subset F_{\text {sol }}^{2}
$$

Combining the equations (29), (30), (35) and (36) one concludes the poof of theorem 3.1. The proof in the discrete case being similar, we will just remind below the ergodic theorem which is used. For any bounded set $K \subset \mathbb{Z}^{d}$. Let $K_{t}=\left\{x \in \mathbb{Z}^{d}, t^{-1} x \in K\right\}$ denote the homothetic dilatation, with ratio $t>0$, of the set $K$. Let $f(x) \in L_{l o c}^{1}\left(\mathbb{Z}^{d}\right)$. A number $M\{f\}$ is called the mean value of $f$ if

$$
\lim _{t \rightarrow \infty} \frac{1}{t^{d}|K|} \sum_{x \in K_{t}} f(x)=M\{f\}
$$

For any bounded set $K \subset \mathbb{Z}^{d}$.

The following theorem is the theorem 9 of the chapter VIII.6.9 of [DS67]

Theorem 3.6. Let $f \in L^{p}(\mu)$ with $1 \leq p<\infty$. Then for almost all $\eta \in X$ the realization $T_{x} f(\eta)$ posses a mean value in the sense of (37). Moreover, the mean value $M\left\{T_{x} f(\eta)\right\}$, considered as a function of $\eta \in X$ is invariant, and for almost all $\eta \in X$

$$
<f>\equiv \int_{X} f(\eta) \mu(d \eta)=M\left\{T_{x} f(\eta)\right\}
$$

If $p>1$, the limit in (37) also exists in the norm of $L^{p}$ and the functions are for $t>0$, all dominated by a function in $L^{p}$.

\subsection{Periodic approximation of variational function- als on the ergodic spaces}

In this subsection it will be shown that the almost sure continuity of Weyl's decomposition has a direct consequence on variational functionals on the ergodic space. The results and notations given in this subsection are valid in the continuous case as in the discrete case. Let $m, p \in \mathbb{N}^{2}$, and

$$
\Phi: \quad\left(\eta, X^{1}, \ldots, X^{m}, Y^{1}, \ldots, Y^{p}\right) \longrightarrow \Phi\left(\eta, X^{1}, \ldots, X^{m}, Y^{1}, \ldots, Y^{p}\right)
$$

a mapping from $X \times \mathbb{R}^{d \times m} \times \mathbb{R}^{d \times p}$ into $\mathbb{R}^{+}$such that for any $\left(\xi^{1}, \ldots, \xi^{m}, \nu^{1}, \ldots, \nu^{p}\right) \in\left(F_{\text {pot }}^{2}\right)^{m} \times\left(F_{\text {sol }}^{2}\right)^{p}$ one has

$$
\eta \rightarrow \Phi\left(\eta, \xi^{1}, \ldots, \xi^{m}, \nu^{1}, \ldots, \nu^{p}\right) \in \mathrm{七}^{1}(X, \mu) .
$$


We write

$$
Z(\Phi, \mu):=\inf _{\left(\xi^{1}, \ldots, \xi^{m}, \nu^{1}, \ldots, \nu^{p}\right) \in\left(F_{p o t}^{2}\right)^{m} \times\left(F_{\text {sol }}^{2}\right)^{p}}\left\langle\Phi\left(\eta, \xi^{1}, \ldots, \xi^{m}, \nu^{1}, \ldots, \nu^{p}\right)\right\rangle
$$

Let us define for $N>0$ the function (in the continuous case)

$$
\begin{aligned}
\Psi_{N}: X \times\left(\mathrm{E}^{2}\left(T_{N}^{d}\right)\right)^{m} \times\left(\mathrm{E}^{2}\left(T_{N}^{d}\right)\right)^{p} & \rightarrow \mathbb{R}^{+} \\
\left(\eta, v^{1}, \ldots, v^{m}, q^{1}, \ldots, q^{p}\right) \rightarrow N^{-d} & \int_{x \in[0, N(d} \Phi\left(\tau_{-x} \eta, v^{1}(x), \ldots, v^{m}(x)\right. \\
& \left., q^{1}(x), \ldots, q^{p}(x)\right) d x
\end{aligned}
$$

In the discrete case we shall write

$$
\begin{aligned}
\Psi_{N}: X \times\left(\mathrm{E}^{2}\left(T_{N}^{d}\right)\right)^{m} \times\left(\mathrm{E}^{2}\left(T_{N}^{d}\right)\right)^{p} & \rightarrow \mathbb{R}^{+} \\
\left(\eta, v^{1}, \ldots, v^{m}, q^{1}, \ldots, q^{p}\right) & \rightarrow N^{-d} \sum_{\substack{x \in \mathbb{Z} \cap[0, N(d\\
}} \Phi\left(q^{1}(x), \ldots, q^{p}(x)\right)
\end{aligned}
$$

Let us define for $N \in \mathbb{N}$ the random variable $Z(N, \eta)$ by

$$
Z(N, \eta):=\inf _{\left(v^{1}, \ldots, v^{m}, q^{1}, \ldots, q^{p}\right) \in\left(F_{\text {pot }}^{2}\left(T_{N}^{d}\right)\right)^{m} \times\left(F_{\text {sol }}^{2}\left(T_{N}^{d}\right)\right)^{p}} \Psi_{N}\left(\eta, v^{1}, \ldots, v^{m}, q^{1}, \ldots, q^{p}\right)
$$

Observe that $Z(N, \eta)$ corresponds to the periodization of the variational problem associated to $Z(\Phi, \mu)$ over the torus $T_{N}^{d}$ for a particular realization $\eta$ of the ergodic space.

Definition 3.7. We say that the function $\Phi$ is admissible if there exists a strictly increasing continuous function $g$ from $\mathbb{R}^{+}$into $\mathbb{R}^{+}$such that for all $\eta \in X$, the function

$$
\begin{aligned}
g \circ \Psi_{N}(\eta):\left(\mathrm{E}^{2}\left(T_{N}^{d}\right)\right)^{m} \times\left(\mathrm{E}^{2}\left(T_{N}^{d}\right)\right)^{p} & \rightarrow \mathbb{R}^{+} \\
\left(v^{1}, \ldots, v^{m}, q^{1}, \ldots, q^{p}\right) & \rightarrow g\left(\Psi_{N}\left(\eta, v^{1}, \ldots, v^{m}, q^{1}, \ldots, q^{p}\right)\right)
\end{aligned}
$$

is upper semi-continuous with respect to the norm $\sum_{i=1}^{m}\left\|v^{i}\right\|_{\mathrm{E}^{2}\left(T_{N}^{d}\right)}+\sum_{j=1}^{p}\left\|v^{j}\right\|_{\mathrm{E}^{2}\left(T_{N}^{d}\right)}$ a.s. uniformly in $N$ and $\eta$.

Theorem 3.8. If the function $\Phi$ is admissible then for $\mu$-almost all $\eta \in X$

$$
\lim \sup _{N \rightarrow \infty} Z(N, \eta) \leq Z(\Phi, \mu)
$$


Proof. We will write the theorem for the continuous case. In the discrete case the proof is trivially similar. Let $\left(\xi^{1}, \ldots, \xi^{m}, \nu^{1}, \ldots, \nu^{p}\right) \in\left(F_{\text {pot }}^{2}\right)^{m} \times$ $\left(F_{\text {sol }}^{2}\right)^{p}$. To prove the theorem it is sufficient to show that for $\mu$-almost all $\eta \in X$

$$
\lim \sup _{N \rightarrow \infty} g(Z(N, \eta)) \leq g\left(\left\langle\Phi\left(\eta, \xi^{1}, \ldots, \xi^{m}, \nu^{1}, \ldots, \nu^{p}\right)\right\rangle\right)
$$

By the equation (43) we have

$$
g(Z(N, \eta))=J_{1}(N)+J_{2}(N)
$$

with

$J_{1}(N)=g\left(N^{-d} \int_{x \in[0, N(d} \Phi\left(\tau_{-x} \eta, \xi^{1}(x, \eta), \ldots, \xi^{m}(x, \eta), \nu^{1}(x, \eta), \ldots, \nu^{p}(x, \eta)\right) d x\right)$

and

$$
\begin{aligned}
J_{2}(N)= & \inf _{\left(v^{1}, \ldots, v^{m}, q^{1}, \ldots, q^{p}\right) \in\left(F_{\text {pot }}^{2}\left(T_{N}^{d}\right)\right)^{m} \times\left(F_{\text {sol }}^{2}\left(T_{N}^{d}\right)\right)^{p}}\left(g\left(\Psi_{N}\left(\eta, v^{1}, \ldots, v^{m}, q^{1}, \ldots, q^{p}\right)\right)\right. \\
& \left.-g\left(\Psi_{N}\left(\eta, \Pi_{N} \xi^{1}, \ldots, \Pi^{N} \xi^{m}, \Pi^{N} \nu^{1}, \ldots, \Pi^{N} \nu^{p}\right)\right)\right)
\end{aligned}
$$

by the ergodic theorem 3.5 one has for $\mu$-almost all $\eta \in X$

$$
\lim _{N \rightarrow \infty} J_{1}(N)=g\left(\left\langle\Phi\left(\eta, \xi^{1}, \ldots, \xi^{m}, \nu^{1}, \ldots, \nu^{p}\right)\right\rangle\right)
$$

Now by theorem 3.1 for $\mu$-almost all $\eta \in X$ there exists sequences $v^{1, N}, \ldots, q^{1, N}, v^{1, N}, \ldots, q^{p, N} \in\left(F_{\text {pot }}^{2}\left(T_{N}^{d}\right)\right)^{m} \times\left(F_{\text {sol }}^{2}\left(T_{N}^{d}\right)\right)^{p}$ such that

$$
\lim _{N \rightarrow \infty} \sum_{i=1}^{m}\left\|\Pi_{N} \xi^{i}(\eta)-v^{i, N}\right\|_{\mathrm{E}^{2}\left(T_{N}^{d}\right)}+\sum_{j=1}^{p}\left\|\Pi_{N} \nu^{j}(\eta)-v^{j, N}\right\|_{\mathrm{E}^{2}\left(T_{N}^{d}\right)}=0
$$

From equation (49) one obtains

$$
\begin{aligned}
J_{2}(N) \leq & g\left(\Psi_{N}\left(\eta, v^{1, N}, \ldots, v^{m, N}, q^{1, N}, \ldots, q^{p, N}\right)\right) \\
& -g\left(\Psi_{N}\left(\eta, \Pi_{N} \xi^{1}, \ldots, \Pi^{N} \xi^{m}, \Pi^{N} \nu^{1}, \ldots, \Pi^{N} \nu^{p}\right)\right)
\end{aligned}
$$

Combining (52) with (51) and the uniform upper semi-continuity of $g \circ \psi(\eta)$ we concludes that for $\mu$-almost all $\eta \in X$

$$
\lim \sup _{N \rightarrow \infty} J_{2}(N) \leq 0
$$

Which concludes the proof of the theorem. 


\section{Application}

\subsection{Symmetric continuous Operator}

\subsubsection{Homogenization in the ergodic medium}

Let $A(\eta)$ be a $d \times d$ bounded symmetric matrix defined on $X\left(A_{i, j} \in\right.$ $\left.L^{\infty}(X, \mu)\right)$ and satisfying the following ellipticity condition

$$
\nu_{1}|\xi|^{2} \leq^{t} \xi A \xi \leq \nu_{2}|\xi|^{2}, \quad \nu_{1}>0
$$

for almost all $\eta \in X$. Realizations $A(x, \eta)=T_{x} A(\eta)$ of this matrix are considered and we are interested in describing the homogenization for almost all $\eta \in X$ of the operator $\nabla_{x} A(x, \eta) \nabla_{x}$.

Consider $\sigma(A, \mu)$ the $d \times d$ positive definite symmetric matrix defined by the following variational formula: for $\xi \in \mathbb{R}^{d}$

$$
{ }^{t} \xi \sigma(A, \mu) \xi=\inf _{v \in F_{p o t}^{2}}\left\langle{ }^{t}(\xi+v) A(\xi+v)\right\rangle
$$

Observe that $\sigma(A, \mu)$ corresponds to the effective conductivity associated to the operator $\nabla_{x} A(x, \eta) \nabla_{x}$. Indeed by the theorem 7.4 of [JKO91] for any bounded domain $Q \subset \mathbb{R}^{d}$ and any $f \in H^{-1}(Q)$ the solutions $u^{\epsilon}$ of the Dirichlet problems $\left(A^{\epsilon}(x, \eta)=A(x / \epsilon, \eta)\right)$

$$
\nabla A^{\epsilon} \nabla u^{\epsilon}=f, \quad u^{\epsilon} \in H_{0}^{1}(Q)
$$

possess the following properties of convergence (in the weak topology)

$$
u^{\epsilon} \rightarrow u^{0} \text { in } H_{0}^{1}(Q), \quad A^{\epsilon} \nabla u^{\epsilon} \rightarrow \sigma(A, \mu) \nabla u^{0} \text { in } \mathrm{七}^{2}(Q)
$$

where $u^{0}$ is the solution of the Dirichlet problem

$$
\nabla \sigma(A, \mu) \nabla u^{0}=f, \quad u^{0} \in H_{0}^{1}(Q)
$$

Moreover writing $y_{t}^{\eta}$ the diffusion associated to the operator $\nabla_{x} A(x, \eta) \nabla_{x}$, and $\mathbb{P}_{\eta}$ the law of that started from 0 in $\mathbb{R}^{d}$ it is well known ( $\mathrm{KV86}$, JKO91], Ol194]) that under the law $\mu \otimes \mathbb{P}_{\eta}, \epsilon y_{t / \epsilon^{2}}^{\eta}$ converges in law as $\epsilon \downarrow 0$ towards a Brownian Motion starting from 0 with covariance matrix (effective diffusivity) $2 \sigma(A)$.

\subsubsection{Periodization of the ergodic medium}

For $\eta \in X$, we write $A^{N}(x, \eta)$ obtained by periodizing $A(x, \eta)$ over the torus $T_{N}^{d}\left(\right.$ of dimension $d$ and side $N, \mathbb{R}^{d} /\left(N \mathbb{Z}^{d}\right)$ )

$$
A^{N}(x, \eta)=A(x-N[x / N], \eta)
$$


where $[y]$ is the integer part of $y$. For $\eta \in X$, we define $\sigma\left(A^{N}, \eta\right)$ the $d \times d$ symmetric positive definite matrix by the following variational formula: for $\xi \in \mathbb{R}^{d}$

$$
{ }^{t} \xi \sigma\left(A^{N}, \eta\right) \xi=\inf _{f \in C^{\infty}\left(T_{N}^{d}\right)} N^{-d} \int_{T_{N}^{d}}{ }^{t}(\xi+\nabla f(x)) A^{N}(x, \eta)(\xi+\nabla f(x)) d x
$$

Observe that $\sigma\left(A^{N}, \eta\right)$ corresponds to the effective conductivity associated to the periodic operator $\nabla A^{N}(x, \eta) \nabla$ in the sense given above in the equations (56), (57) and (57). Writing $y_{t}^{\eta, N}$ the diffusion associated to the operator $\nabla_{x} A^{N}(x, \eta) \nabla_{x}$, it is well known ([JKO91], Oll94]) that $\epsilon y_{t / \epsilon^{2}}^{\eta, N}$ converges in law as $\epsilon \downarrow 0$ towards a Brownian Motion starting from 0 with covariance matrix (effective diffusivity) $2 \sigma\left(A^{N}, \eta\right)$. Notice, whereas $\sigma(A, \mu)$ is a constant (not random) matrix, $\sigma\left(A^{N}, \eta\right)$ is a random matrix on $X$, which depends on the particular realization $A^{N}(x, \eta)$ of the periodic environment.

\subsubsection{The main theorem}

It is our purpose to prove the following theorem

Theorem 4.1. For $\mu$-almost all $\eta \in X$

$$
\lim _{N \rightarrow+\infty} \sigma\left(A^{N}, \eta\right)=\sigma(A, \mu)
$$

\subsubsection{Proof}

Let $\xi \in \mathbb{R}^{d}$. Let us apply theorem 4.1 with $m=1, p=0$ and $\Phi\left(\eta, X^{1}\right)=$ ${ }^{t}\left(\xi+X^{1}\right) A(\eta)\left(\xi+X^{1}\right)$. By the Minkowski inequality and the uniform ellipticity condition (54) one has that for $N \in \mathbb{N}^{*}$ and $\mu$-almost all $\eta \in X$, $v^{1}, v^{2} \in\left(\mathrm{E}^{2}\left(T_{N}^{d}\right)\right)^{2}$

$$
\left(\Psi_{N}\left(\eta, v^{1}\right)\right)^{\frac{1}{2}}-\left(\Psi_{N}\left(\eta, v^{2}\right)\right)^{\frac{1}{2}} \leq \nu_{2}\left\|v^{1}-v^{2}\right\|_{\mathrm{Ł}^{2}\left(T_{N}^{d}\right)}
$$

It follows that $\Phi$ is admissible and from the variational formulae (60), (60) and theorem 4.1 one obtains that for $\mu$-almost all $\eta \in X$

$$
\lim \sup _{N \rightarrow \infty}{ }^{t} \xi \sigma\left(A^{N}, \eta\right) \xi \leq{ }^{t} \xi \sigma(A, \mu) \xi
$$

Which gives the upper bound of theorem 4.1. For the lower bound we will apply theorem 4.1 with $m=0, p=1$ and $\Phi\left(\eta, Y^{1}\right)={ }^{t}\left(\xi+Y^{1}\right) A^{-1}(\eta)\left(\xi+Y^{1}\right)$. By the Minkowski inequality and the uniform ellipticity condition (54) one has that for $N \in \mathbb{N}^{*}$ and $\mu$-almost all $\eta \in X, q^{1}, q^{2} \in\left(\mathrm{E}^{2}\left(T_{N}^{d}\right)\right)^{2}$

$$
\left(\Psi_{N}\left(\eta, q^{1}\right)\right)^{\frac{1}{2}}-\left(\Psi_{N}\left(\eta, q^{2}\right)\right)^{\frac{1}{2}} \leq\left(\nu_{1}\right)^{-1}\left\|q^{1}-q^{2}\right\|_{\complement^{2}\left(T_{N}^{d}\right)}
$$


Moreover let us remind the following well known ([JKO91] variational formulas: for $l \in \mathbb{R}^{d}$

$$
\begin{gathered}
{ }^{t} l \sigma(A, \mu)^{-1} l=\inf _{p \in F_{\text {sol }}^{2}}\left\langle(l+p) A^{-1}(l+p)\right\rangle \\
{ }^{t} l \sigma\left(A^{N}, \eta\right)^{-1} l=\inf _{\nu \in F_{\text {sol }}^{2}\left(T_{N}^{d}\right)} N^{-d} \int_{T_{N}^{d}}{ }^{t}(l+\nu(x))\left(A^{N}(x, \eta)\right)^{-1}(l+\nu(x)) d x
\end{gathered}
$$

Then it follows that $\Phi$ is admissible and from the variational formulae (65), (66) and theorem 4.1 one obtains that for $\mu$-almost all $\eta \in X$

$$
\lim \sup _{N \rightarrow \infty}{ }^{t} l\left(\sigma\left(A^{N}, \eta\right)\right)^{-1} l \leq{ }^{t} l(\sigma(A, \mu))^{-1} l
$$

Which gives the lower bound of theorem 4.1

\subsection{Non symmetric continuous Operator, diffusion in divergence free flow}

\subsubsection{Homogenization in the ergodic medium}

Let $E$ be a $d \times d$ bounded skew-symmetric matrix defined on $X\left(E_{i, j} \in\right.$ $\left.L^{\infty}(X, \mu)\right)$. Let $a$ be a constant symmetric positive definite $d \times d$ matrix. Realizations $E(x, \eta)=T_{x} E(\eta)$ of this matrix are considered and we are interested in describing the homogenization for almost all $\eta \in X$ of the operator

$$
L_{E}=\nabla_{x}(a+E(x, \eta)) \nabla_{x}
$$

$E$ is seen as the stream matrix of the incompressible flow ${ }^{t} \nabla . E$.

Let $z_{t}^{\eta}$ be the process generated by $L_{E}$, and $\mathbb{P}_{\eta}$ the law of that diffusion started from 0 in $\mathbb{R}^{d}$. It is well known (see for instance Oll94)that under the law $\mu \otimes \mathbb{P}_{\eta}$ as $\epsilon \downarrow 0, \epsilon z_{t / \epsilon^{2}}^{\eta}$ converges in law to a Brownian motion with covariance matrix $D(a, E, \mu)$ : for $l \in \mathbb{R}^{d}$

$$
{ }^{t} l D(a, E, \mu) l=2^{t} l a l+2\left\langle\left|v_{l}\right|_{a}^{2}\right\rangle
$$

Where we have used the notation $|\xi|_{a}^{2}:={ }^{t} \xi a \xi$ for $\xi \in \mathbb{R}^{d}$ and $v_{l}$ defined as the unique solution $u \in F_{p o t}^{2}$ of

$$
<\phi .(a+E)(l+u)>=0, \quad \forall \phi \in F_{p o t}^{2} ; \quad u \in F_{p o t}^{2}
$$

The existence of a solution for this problem follows from the Lax-Milgram Lemma and the estimate $\left\langle v \cdot(a+E) v>\geq \lambda_{\min }(a)\|v\|_{\mathrm{L}^{2}(X, \mu)}^{2}\right.$; JKO91].

Obviously, the solution $v_{l}$ of the problem (70) depends linearly on $l \in \mathbb{R}^{d}$. 
Therefore

$\left\langle(a+E)\left(l+v_{l}\right)\right\rangle$ is a linear form with respect to $l$. The effective conductivity $\sigma(a, E, \mu)$ is defined by

$$
\sigma(a, E, \mu) l=\left\langle(a+E)\left(l+v_{l}\right)\right\rangle
$$

It is a non-symmetric matrix relating the gradient of the heat intensity with the flux FP94 by (71). Observe that the symmetric part of the effective conductivity gives the effective diffusivity by the following relation:

$$
D(a, E, \mu)=2 \sigma_{\mathrm{sym}}(a, E)
$$

\subsubsection{Periodization of the ergodic medium}

For $\eta \in X$, we write $E^{N}(x, \eta)$ obtained by periodizing $E(x, \eta)$ over the torus $T_{N}^{d}$

$$
E^{N}(x, \eta)=E(x-N[x / N], \eta)
$$

We are interested in describing the homogenization for almost all $\eta \in X$ of the operator

$$
L_{E}^{N}=\nabla_{x}\left(a+E^{N}(x, \eta)\right) \nabla_{x}
$$

Let $z_{t}^{\eta, N}$ be the process generated by $L_{E}^{N}$. It is well known (see for instance Nor97])that as $\epsilon \downarrow 0, \epsilon z_{t / \epsilon^{2}}^{\eta, N}$ converges in law to a Brownian motion with covariance matrix $D\left(a, E^{N}, \eta\right)$ with for $l \in \mathbb{R}^{d}$

$$
{ }^{t} l D\left(a, E^{N}, \eta\right) l=2^{t} l a l+2 N^{-d} \int_{T_{N}^{d}}\left|\psi_{l}(x, \eta)\right|_{a}^{2} d x
$$

Where $\psi_{l}$ defined as the unique solution $\psi \in H^{1}\left(T_{N}^{d}\right)$ of

$$
\int_{T_{N}^{d}} \phi(x)\left(a+E^{N}(x, \eta)\right)(l+\psi(x)) d x=0, \quad \forall \phi \in H^{1}\left(T_{N}^{d}\right) ; \quad \psi \in H^{1}\left(T_{N}^{d}\right)
$$

We have noted $H^{1}\left(T_{N}^{d}\right)$ the closure of $\left\{\nabla f: f \in C^{\infty}\left(T_{N}^{d}\right)\right\}$ in $L^{2}\left(T_{N}^{d}\right)$ with respect to the $L^{2}$-norm. Obviously, the solution $\psi_{l}$ of the problem (76) depends linearly on $l \in \mathbb{R}^{d}$. Therefore $\int_{T_{N}^{d}}\left(a+E^{\eta, N}(x)\right)\left(l+\psi_{l}(x, \eta)\right) d x$ is a linear form with respect to $l$. The effective conductivity $\sigma\left(a, E^{N}, \eta\right)$ is defined by: for $l \in \mathbb{R}^{d}$

$$
\sigma\left(a, E^{N}, \eta\right) l=\int_{T_{N}^{d}}\left(a+E^{\eta, N}(x)\right)\left(l+\psi_{l}(x)\right) d x
$$

It is a non-symmetric matrix relating the gradient of the heat intensity with the flux [FP94 by (77). Observe that the symmetric part of the effective conductivity gives the effective diffusivity by the following relation:

$$
D\left(a, E^{N}, \eta\right)=2 \sigma_{\mathrm{sym}}\left(a, E^{N}, \eta\right)
$$




\subsubsection{The main theorem}

It is our purpose to prove the following theorem

Theorem 4.2. For $\mu$-almost all $\eta \in X$

$$
\lim _{N \rightarrow+\infty} \sigma\left(a, E^{N}, \eta\right)=\sigma(a, E, \mu)
$$

In particular

$$
\lim _{N \rightarrow+\infty} D\left(a, E^{N}, \eta\right)=D(a, E, \mu)
$$

\subsubsection{Core of the proof: Variational formulations and theo- rem 4.1}

As for a symmetric operator, the proof theorem 4.2 relies theorem 3.1 and the variational formulae associated to the effective conductivity.

4.2.4.1 Variational Formulation of the effective diffusivity in the periodic case For $N>0$, let us write $\mathcal{S}\left(T_{N}^{d}\right)$ the set of skewsymmetric matrices with smooth coefficients defined on $T_{N}^{d}$ and for $H \in$ $\mathcal{S}\left(T_{N}^{d}\right), \operatorname{div} H$ is the vector field defined by $(\operatorname{div} H)_{i}=\sum_{j=1}^{d} \partial_{j} H_{i, j}$.

In the periodic case, we will use Norris's variational formulation (obtained by polarization [Nor97]) to control $\sigma\left(a, E^{N}, \eta\right)$. For $y \in \mathbb{R}^{d}$ we will write $|y|_{a^{-1}}^{2}:={ }^{t} y a^{-1} y$.

For all $\xi, l \in \mathbb{R}^{d}$,

$$
\begin{aligned}
& \left|\xi-\sigma\left(a, E^{N}, \eta\right) l\right|_{\sigma_{\mathrm{sym}}^{-1}\left(a, E^{N}, \eta\right)}^{2} \\
& \inf _{f, H \in C^{\infty}\left(T_{N}^{d}\right) \times \mathcal{S}\left(T_{N}^{d}\right)} N^{-d} \int_{T_{N}^{d}}\left|\xi-\nabla H-\left(a+E^{N}(x, \eta)\right)(l-\nabla f)\right|_{a^{-1}}^{2} d x
\end{aligned}
$$

For all $l \in \mathbb{R}^{d}$

$|l|_{\sigma_{\mathrm{sym}}\left(a, E^{N}, \eta\right)}^{2}=\inf _{\xi \perp l, f, H \in C^{\infty}\left(T_{N}^{d}\right) \times \mathcal{S}\left(T_{N}^{d}\right)} N^{-d} \int_{T_{N}^{d}}\left|\xi-\nabla H-\left(a+E^{N}(x, \eta)\right)(l-\nabla f)\right|_{a^{-1}}^{2} d x$

Where we have written $\xi \perp l:=\left\{\xi \in \mathbb{R}^{d}: \xi . l=0\right\}$. We also have for all $\xi \in \mathbb{R}^{d}$

$|\xi|_{\sigma_{\text {sym }}^{-1}\left(a, E^{N}, \eta\right)}^{2}=\inf _{f, H \in C^{\infty}\left(T_{N}^{d}\right) \times \mathcal{S}\left(T_{N}^{d}\right)} N^{-d} \int_{T_{N}^{d}}\left|\xi-\nabla H+\left(a+E^{N}(x, \eta)\right) \nabla f\right|_{a^{-1}}^{2} d x$ 
Remark 4.3. Let us remind, as it has been noticed by J.R. Norris [Nor97, that from (82) and (83) one obtains that

$$
a \leq \sigma_{\mathrm{sym}}\left(a, E^{N}, \eta\right) \leq a+N^{-d} \int_{T_{N}^{d}}{ }^{t} E^{N}(x, \eta) a^{-1} E^{N}(x, \eta) d x
$$

For a saddle point variational formulation we refer to [FP94].

\subsubsection{Variational Formulation of the effective diffusivity} in the ergodic case The following theorem proven in subsection 5.2.1 is inspired from the variational formulation given for the periodic case by J. R. Norris [Nor97] (lemma 3.1), (for a non local variational formulation we refer to [FP96])

Theorem 4.4. For all $\xi, l \in \mathbb{R}^{d}$,

$$
|\xi-\sigma(a, E, \mu) l|_{\sigma_{\text {sym }}^{-1}(a, E, \mu)}^{2}=\inf _{v, p \in F_{p o t} \times F_{s o l}}\left\langle|\xi-p-(a+E)(l-v)|_{a^{-1}}^{2}\right\rangle
$$

for $l \in \mathbb{R}^{d}$

$$
{ }^{t} l \sigma_{\mathrm{sym}}(a, E, \mu) l=\inf _{\xi \perp l, v \in F_{p o t}^{2}, p \in F_{\text {sol }}^{2}}\left\langle|\xi-p-(a+E)(l-v)|_{a^{-1}}^{2}\right\rangle
$$

For all $\xi \in \mathbb{R}^{d}$

$$
|\xi|_{\sigma_{\text {sym }}^{-1}(a, E, \mu)}^{2}=\inf _{v, p \in F_{p o t} \times F_{\text {sol }}}\left\langle|\xi-p+(a+E) v|_{a^{-1}}^{2}\right\rangle
$$

Remark 4.5. Let us observe that from (86) and (87) one obtains that

$$
a \leq \sigma_{\mathrm{sym}}(a, E, \mu) \leq a+\left\langle{ }^{t} E a^{-1} E\right\rangle
$$

Let $\xi, l \in \mathbb{R}^{d}$. Let us apply theorem 4.1 with $m=1, p=1$ and $\Phi\left(\eta, X^{1}, Y^{1}\right)=\left|\xi-Y^{1}-(a+E)\left(l-X^{1}\right)\right|_{a^{-1}}^{2}$. By the Minkowski inequality one has that for $N \in \mathbb{N}^{*}$ and $\mu$-almost all $\eta \in X, v^{1}, v^{2}, q^{1}, q^{2} \in\left(\mathrm{E}^{2}\left(T_{N}^{d}\right)\right)^{4}$

$$
\begin{aligned}
\left(\Psi_{N}\left(\eta, v^{1}, q^{1}\right)\right)^{\frac{1}{2}} & -\left(\Psi_{N}\left(\eta, v^{2}, q^{2}\right)\right)^{\frac{1}{2}} \leq C_{d}\left(\lambda_{\min }(a)\right)^{-1 / 2}\left\|q^{1}-q^{2}\right\|_{\mathrm{E}^{2}\left(T_{N}^{d}\right)} \\
& +C_{d}\left(\lambda_{\max }(a)+\|E\|_{L^{\infty}(X, \mu)}\right)\left(\lambda_{\min }(a)\right)^{-1 / 2}\left\|v^{1}-v^{2}\right\|_{\mathrm{Ł}^{2}\left(T_{N}^{d}\right)}
\end{aligned}
$$

It follows that $\Phi$ is admissible and from the variational formulae (85), (81) and theorem 4.1 one obtains that for $\mu$-almost all $\eta \in X$

$$
\lim \sup _{N \rightarrow \infty}\left|\xi-\sigma\left(a, E^{N}(\eta)\right) l\right|_{\sigma_{\text {sym }}^{-1}\left(a, E^{N}, \eta\right)}^{2} \leq|\xi-\sigma(a, E, \mu) l|_{\sigma_{\text {sym }}^{-1}(a, E, \mu)}^{2}
$$


Choosing $\xi:=\sigma(a, E, \mu) l$ in this equation, one obtains from (84) and (88) that for $\mu$-almost all $\eta \in X$

$$
\lim _{N \rightarrow \infty} \sigma\left(a, E^{N}(\eta)\right) l=\sigma(a, E, \mu) l
$$

Which concludes the proof of theorem 4.2 .

\subsection{Discrete Operator}

We shall extend in this subsection our results to the discrete case.

\subsubsection{The ergodic homogenization problem}

We will consider a symmetric random walk on $\mathbb{Z}^{d}$ as in CI01 but with ergodic jump rates instead of i.i.d. The random ergodic environment will be represented by the random $d$-dimensional vector $\xi_{i}(\eta)(i \in\{1, \ldots, d\})$ on $X$, we will write $\xi_{i}(x, \eta)=\xi_{i}\left(\tau_{-x} \eta\right)$. We will assume that there exists $c \geq 1$ such that for $\mu$-almost all $\eta \in X$,

$$
1 / c \leq \xi_{i}(\eta) \leq c
$$

Let us write $X(t, \xi(\eta))$ the nearest neighbor symmetric random walk on $\mathbb{Z}^{d}$ with jump according to $\xi_{i}(x, \eta)$ rates $\left(\xi_{i}(x, \eta)\right.$ is the jump rate between from the site $x$ to the site $x+e_{i}$ and also from the site $x+e_{i}$ to the site $x$ ).

In the quenched regime (for a fixed $\eta$ ), $\mathbb{P}_{x}^{\xi(\eta)}$ stands for the probability law of this process when the walk starts at $x \in \mathbb{Z}^{d}$. It is well known ( $\mathrm{KV} 86$, MFGW89, CI01]) that in the annealed regime (under the law $\mu \otimes \mathbb{P}_{0}^{\xi(\eta)}$ ) as $\epsilon \downarrow 0, \epsilon X\left(t / \epsilon^{2}, \xi(\eta)\right)$ converges in law towards a Brownian Motion with covariance matrix (effective diffusivity) $D(\xi, \mu)$.

\subsubsection{Periodization of the ergodic medium}

For $N \in \mathbb{N}^{*}$ and $\eta \in X$ we write $\xi^{N}(\eta)$ the periodized bond configuration associated to $\xi(x, \eta)$ over the torus $T_{N}=\mathbb{Z}^{d} / N \mathbb{Z}^{d}$. For $x \in \mathbb{Z}^{d}$ decomposed as $x=y+N z$ with $y \in\{0, \ldots, N-1\}^{d}$ and $z \in \mathbb{Z}^{d}$ we define $\xi^{N}(x, \eta)$ by

$$
\xi^{N}(x, \eta):=\xi(y, \eta)
$$

It is well known ( $\mathrm{CI01}$ ) that in the quenched regime (under the law $\mathbb{P}_{0}^{\xi^{N}(\eta)}$ ) as $\epsilon \downarrow 0, \epsilon X\left(t / \epsilon^{2}, \xi^{N}(\eta)\right)$ converges in law towards a Brownian Motion on $\mathbb{Z}^{d}$ with covariance matrix (effective diffusivity) $\sigma\left(\xi^{N}, \eta\right)$ (which is a random matrix on $X$, depending on the particular realization $\xi^{N}(\eta)$ ). 


\subsubsection{The main theorem}

It is our purpose to prove the following theorem

Theorem 4.6. For $\mu$-almost all $\eta \in X$

$$
\lim _{N \rightarrow+\infty} \sigma\left(\xi^{N}, \eta\right)=\sigma(\xi, \mu)
$$

This result has already been given in [CI01] when the jump rates are i.i.d. It is interesting to note that when the jump rates are i.i.d., D. Ioffe and P. Caputo have shown an exponential rate of convergence of $\sigma\left(\xi^{N}, \eta\right)$ towards $\mathbb{E}_{\mu}\left[\sigma\left(\xi^{N}, \eta\right)\right]$ as $N \rightarrow \infty$.

\subsubsection{Proof}

We shall use the variational formula given in CI01: for $l \in \mathbb{R}^{d}$

$$
{ }^{t} l \sigma(\xi, \mu) l=\inf _{f \in L^{2}(\mu)} \sum_{i=1}^{d}\left\langle\xi_{i}\left(l_{i}+D_{i} f\right)^{2}\right\rangle
$$

Let us also remind the variational formula

$$
{ }^{t} l \sigma\left(\xi^{N}, \eta\right) l=\inf _{f \in L^{2}\left(T_{N}^{d}\right)}\left|T_{N}^{d}\right|^{-1} \sum_{x \in T_{N}^{d}} \sum_{i=1}^{d} \xi_{i}^{N}(x, \eta)\left(l_{i}+\nabla_{i} f(x)\right)^{2}
$$

We will prove in the subsection 5.2 .3 the following lemma which corresponds to the variational formulation of $D^{-1}(\xi)$

Lemma 4.7. For $l \in \mathbb{R}^{d}$

$$
{ }^{t} l \sigma(\xi, \mu)^{-1} l=\inf _{H \in \mathcal{S}(X, \mu)} \sum_{i=1}^{d}\left\langle\xi_{i}^{-1}\left(l_{i}+(\mathcal{D i v} H)_{i}\right)^{2}\right\rangle
$$

It is also easy to prove that for $l \in \mathbb{R}^{d}$ (the proof is similar to the one of lemma 97.)

$$
{ }^{t} l \sigma\left(\xi^{N}, \eta\right)^{-1} l=\inf _{H \in \mathcal{S}\left(T_{N}^{d}\right)} N^{-d} \sum_{x \in T_{N}^{d}} \sum_{i=1}^{d} \xi_{i}^{N}(x, \eta)^{-1}\left(l_{i}+(\operatorname{div} H)_{i}\right)^{2}
$$

Let $l \in \mathbb{R}^{d}$.Let us apply theorem 4.1 with $m=1, p=0$ and $\Phi\left(\eta, X^{1}\right)=$ $\sum_{i=1}^{d} \xi_{i}(\eta)\left(l_{i}+X_{i}^{1}\right)^{2}$. By the Minkowski inequality and the uniform ellipticity condition (92) one has that for $N \in \mathbb{N}^{*}$ and $\mu$-almost all $\eta \in X$, $v^{1}, v^{2} \in\left(\mathrm{Ł}^{2}\left(T_{N}^{d}\right)\right)^{2}$

$$
\left(\Psi_{N}\left(\eta, v^{1}\right)\right)^{\frac{1}{2}}-\left(\Psi_{N}\left(\eta, v^{2}\right)\right)^{\frac{1}{2}} \leq C\left\|v^{1}-v^{2}\right\|_{\mathrm{E}^{2}\left(T_{N}^{d}\right)}
$$


It follows that $\Phi$ is admissible and from the variational formulae (95), (96) and theorem 4.1 one obtains that for $\mu$-almost all $\eta \in X$

$$
\lim \sup _{N \rightarrow \infty}{ }^{t} l \sigma\left(\xi^{N}, \eta\right) l \leq{ }^{t} l \sigma(\xi, \mu) l
$$

Which gives the upper bound of theorem 4.6. The proof of the lower bound is trivially similar: using Minkowski inequality, the uniform ellipticity condition (92), variational formulae (97), (98) and theorem 4.1 one obtains that for $\mu$-almost all $\eta \in X$

$$
\lim \sup _{N \rightarrow \infty}{ }^{t} l\left(\sigma\left(\xi^{N}, \eta\right)\right)^{-1} l \leq{ }^{t} l(\sigma(\xi, \mu))^{-1} l
$$

Which gives the lower bound of theorem 4.6.

\section{Proofs}

\subsection{Main results}

\subsubsection{Proof of theorem 2.6}

It is trivial to check that $F_{\text {pot }}^{2}, F_{\text {sol }}^{2}$ and $\mathbb{R}^{d}$ are mutually orthogonal. Thus in order to prove the Weyl decomposition (2.6) it is sufficient to check that any element of $\mathrm{E}^{2}(X, \mu)$ orthogonal to $F_{\text {pot }}^{2}$ and $\mathbb{R}^{d}$ is an element of $F_{\text {sol }}^{2}$. Let $P$ be an element of $\mathrm{E}^{2}(X, \mu)$ orthogonal to $F_{\text {pot }}^{2}$ and $\mathbb{R}^{d}$. Since $P \perp F_{\text {pot }}^{2}$ it must verify

$$
\sum_{i=1}^{d} D_{i}^{*} P=0
$$

By Lax-Milgram lemma for $m, n \in\{1, \ldots, d\}$, there exists $B^{m, n} \in F_{\text {pot }}^{2}$ such that

$$
\sum_{i=1}^{d} D_{i}^{*} B_{i}^{m, n}=D_{m}^{*} P_{n}
$$

Let us define for $i, m, n \in\{1, \ldots, d\}$

$$
H_{i, n, m}=B_{i}^{m, n}-B_{i}^{n, m}
$$

Let us define $Q \in \mathrm{E}^{2}(X, \mu)$ by for $n \in\{1, \ldots, d\}$

$$
Q_{n}=\sum_{i=1}^{d} H_{i, n, i}
$$

Since $B^{m, n} \in F_{\text {pot }}^{2}$ they can be approximated by gradient forms in $\mathrm{E}_{\text {pot }}^{2}$ and it is easy to deduce that $Q_{n} \in F_{\text {sol }}^{2}$. Moreover for all $n \in\{1, \ldots, d\}$

$$
\sum_{k=1}^{d} D_{k}^{*} D_{k} Q_{n}=\sum_{k=1}^{d} \sum_{i=1}^{d}\left(D_{k}^{*} D_{k} B_{i}^{i, n}-D_{k}^{*} D_{k} B_{i}^{n, i}\right)
$$


Since $B^{m, n} \in F_{\text {pot }}^{2}$ it is easy to check by density that $\eta$-a.s., $D_{k} B_{i}^{i, n}=D_{i} B_{k}^{i, n}$ and $D_{k} B_{i}^{n, i}=D_{i} B_{k}^{n, i}$ thus from the equation (103) one obtains that $\eta$-a.s.

$$
\begin{aligned}
\sum_{k=1}^{d} D_{k}^{*} D_{k} Q_{n} & =\sum_{i=1}^{d} D_{i} \sum_{k=1}^{d}\left(D_{k}^{*} B_{k}^{i, n}(\eta)-D_{k}^{*} B_{k}^{n, i}(\eta)\right) \\
& =\sum_{i=1}^{d} D_{i}\left(D_{i}^{*} P_{n}-D_{n}^{*} P_{i}\right)=\sum_{i=1}^{d} D_{i}^{*} D_{i} P_{n}-D_{n} \sum_{i=1}^{d} D_{i}^{*} P_{i}
\end{aligned}
$$

Using $\sum_{i=1}^{d} D_{i}^{*} P_{i}=0$ we obtain that $\eta$-a.s.

$$
\sum_{k=1}^{d} D_{k}^{*} D_{k}\left(Q_{n}-P_{n}\right)=0
$$

Combining this with $<Q_{n}-P_{n}>=0$, it follows by Lax Milgram lemma that $\eta-$ a.s., $Q_{n}=P_{n}$ and since $Q \in F_{\text {sol }}^{2}$ it follows that $P \in F_{\text {sol }}^{2}$ which concludes the proof of theorem 2.6.

\subsubsection{Proof of equation (22) of lemma 3.3 in the discrete case}

Let $p \in F_{\text {sol }}^{2}$. We will prove in this subsection that for $\mu$-almost all $\eta \in X$

$$
\lim _{N \rightarrow \infty}\left\|\Pi_{N} p(\eta)-\left(\Pi_{N} p(\eta)\right)_{s o l}\right\|_{\mathrm{E}^{2}\left(T_{N}^{d}\right)}=0
$$

For $A \subset \mathbb{R}^{d}$ and $f \in L_{l o c}^{2}\left(\mathbb{Z}^{d}\right)$ we will write

$$
\|f\|_{L^{2}(A)}=\left(\sum_{x \in \mathbb{Z}^{d} \cap A} f(x)^{2}\right)^{\frac{1}{2}}
$$

Observe that to prove the equation (109), it is sufficient to prove the following lemma

Lemma 5.1. For $\mu$-almost all $\eta \in X$, there exists a sequence $\left(K^{N}(x, \eta, M)\right)_{M, N \in \mathbb{N}}$ of skew symmetric matrices with coefficients in $L^{2}\left(T_{N}^{d}\right)$ and a sequence of positive reals $h(M)$ such that $\lim _{M \rightarrow \infty} h(M)=0$ and for $M \geq 10$

$$
\lim \sup _{N \rightarrow \infty} N^{-d / 2}\left\|p(x, \eta)-\operatorname{div} K^{N}(x, \eta, M)\right\|_{L^{2}\left(\left[0, N\left(^{d}\right)\right.\right.} \leq h(M)
$$

Let us now prove lemma [5.1 Let $M \in \mathbb{N}, M \geq 10, M \leq 10^{3} N$. Since $p \in F_{\text {sol }}^{2}$, on obtains from Weyl decomposition (11) that for each $M$, there exists a $d \times d$ skew symmetric matrix $H^{M}$, with coefficients $H_{i, j}^{M} \in L^{2}(\mu)$, $(i, j) \in\{1, \ldots d\}^{2}$ such that $H_{i, j}^{M}=-H_{j, i}^{M}$ and

$$
\sum_{i=1}^{d}\left\langle\left|p-\operatorname{div} H^{M}\right|^{2}\right\rangle \leq 1 / M^{2}
$$


and it is easy to check from the proof of (11) given in subsection 5.1.1 that one can choose $H^{M}$ such that for all $(i, j) \in\{1, \ldots d\}^{2}$

$$
\left\langle\left|\nabla H_{i, j}^{M}\right|^{2}\right\rangle \leq C_{d}\left\langle|p|^{2}\right\rangle
$$

Observe that by the ergodic theorem 3.6] $\eta$-a.s.

$$
\lim \sup _{N \rightarrow \infty}\left(N^{-d} \sum_{x \in \mathbb{Z}^{d} \cap\left[0, N\left(^{d}\right.\right.}\left|p(x, \eta)-\operatorname{div} H^{M}(x, \eta)\right|^{2}\right)^{\frac{1}{2}} \leq 1 / M
$$

Let $g$ be a smooth increasing function on $\mathbb{R}$ such that $g(z)=1$ for $z \geq 1$ and $g(z)=0$ for $z \leq 1 / 2$ and let for $x \in[0,1]^{d} \alpha_{M}(x)=g\left(M \operatorname{dist}\left(x,\left([0,1]^{d}\right)^{c}\right)\right)$. Our candidate for $K^{N}$ will be the skew symmetric $T_{N}^{d}$-periodic matrix:

$$
K^{N}(x, \eta, M)=\left(H^{M}(x, \eta)-[N / M]^{-d} \sum_{y \in\left[0, N / M\left({ }^{d} \cap \mathbb{Z}^{d}\right.\right.} H^{M}(y, \eta)\right) \alpha_{M}(x / N) \quad \text { on } \quad\left[0, N\left({ }^{d} \cap \mathbb{Z}^{d}\right.\right.
$$

Observe that $\alpha_{M}(x)$ is null on an open neighborhood of $\mathbb{R}^{d}$ containing the boundary of $[0,1]^{d}$ and the coefficients of $K^{N}$ can be defined as elements of $\mathrm{E}^{2}\left(T_{N}^{d}\right)$. Let us write

$$
J_{1}(N, M, \eta)=N^{-d / 2}\left\|\operatorname{div} H^{M}(x, \eta)-\operatorname{div} K^{N}(x, \eta, M)\right\|_{L^{2}\left(\left[0, N\left(^{d}\right)\right.\right.}
$$

Observe that $\eta$-a.s. $\left(\nabla \alpha_{M}\right.$ standing for the discrete gradient of $\left.\alpha_{M}\right)$

$$
\begin{aligned}
\operatorname{div} K^{N}(x, \eta, M)= & \operatorname{div} H^{M}(x, \eta) \alpha_{M}(x / N) \\
& +\left(H^{M}(x, \eta)-[N / M]^{-d} \sum_{y \in\left[0, N / M\left(d \cap \mathbb{Z}^{d}\right.\right.} H^{M}(y, \eta)\right) \nabla\left(\alpha_{M}(x / N)\right)
\end{aligned}
$$

Thus

$$
J_{1}(N, M, \eta) \leq J_{2}(N, M, \eta)+J_{3}(N, M, \eta)
$$

with

$$
J_{2}(N, M, \eta)=N^{-d / 2}\left\|\operatorname{div} H^{M}(x, \eta)\left(1-\alpha_{M}(x / N)\right)\right\|_{L^{2}\left(\left[0, N\left(^{d}\right)\right.\right.}
$$

and

$J_{3}(N, M, \eta)=N^{-d / 2}\left\|\left(H^{M}(x, \eta)-[N / M]^{-d} \sum_{y \in\left[0, N / M\left({ }^{d} \cap \mathbb{Z}^{d}\right.\right.} H^{M}(y, \eta)\right) \nabla\left(\alpha_{M}(x / N)\right)\right\|_{L^{2}([0, N(d)}$

Write

$$
A_{M}=\left\{x \in \left[0, N\left(^{d} \cap \mathbb{Z}^{d}: \min _{j \in\{1, \ldots d\}} \min \left(x_{j}, N-x_{j}\right)<N / M\right\}\right.\right.
$$


observe that

$$
\begin{aligned}
J_{2}(N, M, \eta) & \leq N^{-d / 2}\left\|\operatorname{div} H^{M}(x, \eta)\right\|_{L^{2}\left(A_{M}\right)} \\
& \leq C_{d} M^{-\frac{1}{2}}\left(\operatorname{Vol}\left(A_{M}\right)\right)^{-1 / 2}\left\|\operatorname{div} H^{M}(x, \eta)\right\|_{L^{2}\left(A_{M}\right)}
\end{aligned}
$$

and by the ergodic theorem 3.5 ,a.s.

$\left(\operatorname{Vol}\left(A_{M}\right)\right)^{-1 / 2}\left\|\operatorname{div} H^{M}(x, \eta)\right\|_{L^{2}\left(A_{M}\right)} \rightarrow\left\langle\left(\operatorname{div} H^{M}\right)^{2}\right\rangle^{\frac{1}{2}}$ as $N \rightarrow \infty$. Thus $\eta$-a.s.

$$
\lim \sup _{N \rightarrow \infty} J_{2}(N, M, \eta) \leq C_{d} M^{-\frac{1}{2}}\left\langle p^{2}\right\rangle^{\frac{1}{2}}
$$

Now let us prove that

$$
\lim _{M \rightarrow \infty} \lim \sup _{N \rightarrow \infty} J_{3}(N, M, \eta)=0
$$

Since $\left|\nabla\left(\alpha_{M}(x / N)\right)\right| \leq C_{d} M / N$ one has

$J_{3}(N, M, \eta) \leq C_{d} N^{-1-d / 2} M \sum_{m, n}\left\|H_{m, n}^{M}(x, \eta)-[N / M]^{-d} \sum_{y \in\left[0, N / M\left(d \cap \mathbb{Z}^{d}\right.\right.} H_{m, n}^{M}(y, \eta)\right\|_{L^{2}\left(A_{M}\right)}$

Let $I(M)=\left\{\left(i_{1}, \ldots, i_{d}\right) \in\{1, \ldots, M\}^{d} ; \min _{j} \min \left(i_{j}-1, M-i_{j}\right)=0\right\}$ and write $\left\{B_{i}\right\}_{i \in I(M)}$ the set of cubes covering $A_{M}$ (the $N / M$-neighborhood of the border of $\left[0, N\left(^{d}\right)\right.$. More precisely for $i \in I(M)$,

$$
B_{i}=\left\{x \in \left[0, N\left({ }^{d} \cap \mathbb{Z}^{d}: \max _{j}\left|x_{j} / N-\left(i_{j}-0.5\right) / M\right| \leq 1 /(2 M)\right\}\right.\right.
$$

By the equation (124) one has

$$
J_{3}(N, M, \eta)^{2} \leq C_{d} M^{2} \sum_{i \in I(M)} \sum_{m, n} K_{i}^{m, n}
$$

with

$$
K_{i}^{m, n}=N^{-2-d}\left\|H_{m, n}^{M}(x, \eta)-[N / M]^{-d} \sum_{y \in\left[0, N / M\left(d \cap \mathbb{Z}^{d}\right.\right.} H_{m, n}^{M}(y, \eta)\right\|_{L^{2}\left(B_{i}\right)}^{2}
$$

Now using the inequality $(X+Y)^{2} \leq 2 X^{2}+2 Y^{2}$ observe that

$$
\begin{aligned}
K_{i}^{m, n} \leq & \left.2 N^{-2-d} \| H_{m, n}^{M}(x, \eta)-\operatorname{Vol}\left(B_{i}\right)\right)^{-1} \sum_{y \in B_{i}} H_{m, n}^{M}(y, \eta) \|_{L^{2}\left(B_{i}\right)}^{2} \\
& +2 N^{-2} M^{-d}\left([N / M]^{-d} \sum_{y \in\left[0, N / M\left({ }^{d} \cap \mathbb{Z}^{d}\right.\right.} H_{m, n}^{M}(y, \eta)-\left(\operatorname{Vol}\left(B_{i}\right)\right)^{-1} \sum_{y \in B_{i}} H_{m, n}^{M}(y, \eta)\right)^{2}
\end{aligned}
$$


By the Poincaré inequality one has

$$
\left.N^{-2-d} \| H_{m, n}^{M}(x, \eta)-\operatorname{Vol}\left(B_{i}\right)\right)^{-1} \sum_{y \in B_{i}} H_{m, n}^{M}(y, \eta)\left\|_{L^{2}\left(B_{i}\right)}^{2} \leq C_{d} M^{-2} N^{-d}\right\| \nabla H_{m, n}^{M}(x, \eta) \|_{L^{2}\left(B_{i}\right)}^{2}
$$

Thus

$$
\begin{gathered}
J_{3}(N, M, \eta)^{2} \leq C_{d} M^{-1} \sum_{m, n}\left(\operatorname{Vol}\left(A_{M}\right)\right)^{-1}\left\|\nabla H_{m, n}^{M}(x, \eta)\right\|_{L^{2}\left(A_{M}\right)}^{2} \\
+C_{d} M^{2-d} \sum_{m, n} \sum_{i \in I(M)} N^{-2}\left([N / M]^{-d} \sum_{y \in\left[0, N / M\left(d \cap \mathbb{Z}^{d}\right.\right.} H_{m, n}^{M}(y, \eta)-\left(\operatorname{Vol}\left(B_{i}\right)\right)^{-1} \sum_{y \in B_{i}} H_{m, n}^{M}(y, \eta)\right)^{2}
\end{gathered}
$$

But by the ergodic theorem 3.6. $\eta-$ a.s. for all $i \in I(M)$

$$
\begin{aligned}
\lim _{N \rightarrow \infty}\left([N / M]^{-d} \sum_{y \in\left[0, N / M\left(d \cap \mathbb{Z}^{d}\right.\right.} H_{m, n}^{M}(y, \eta)\right. & \left.-\left(\operatorname{Vol}\left(B_{i}\right)\right)^{-1} \sum_{y \in B_{i}} H_{m, n}^{M}(y, \eta)\right) \\
& =<H_{m, n}^{M}>-<H_{m, n}^{M}>=0
\end{aligned}
$$

It is important to observe that $H_{m, n}^{M} \in L^{2}(\mu)$ is sufficient to apply the ergodic theorem 3.6] in order to obtain (131). In the continuous we will not consider an approximation of $p$ but its direct primitive which is not in $L^{2}(\mu)$ explaining why the ergodic theorem will not be applied directly.

It follows that (using (113))

$$
\begin{aligned}
\lim \sup _{N \rightarrow \infty} J_{3}(N, M, \eta)^{2} & \leq C_{d} M^{-1} \sum_{m, n}<\left(\nabla H_{m, n}^{M}\right)^{2}> \\
& \leq C_{d} M^{-1}<p^{2}>
\end{aligned}
$$

And taking the limit $M \rightarrow \infty$ one obtains the equation (123). Now combining equations (112), (116), (117), (122) and (123) one obtains lemma 5.2 .

\subsubsection{Proof of equation (21) of lemma 3.3 in the discrete case}

The proof of equation (21) being similar to the one of equation (22) we will just give its idea. Let $v \in F_{\text {pot }}^{2}$. We have to prove that for $\mu$-almost all $\eta \in X$

$$
\lim _{N \rightarrow \infty}\left\|\Pi_{N} v(\eta)-\left(\Pi_{N} v(\eta)\right)_{p o t}\right\|_{\complement^{2}\left(T_{N}^{d}\right)}=0
$$

Observe that to prove the equation (133) it is sufficient to prove the following lemma 
Lemma 5.2. For $\mu$-almost all $\eta \in X$, there exists a sequence $\left(G^{N}(x, \eta, M)\right)_{M, N \in \mathbb{N}}$ of functions in $E^{2}\left(T_{N}^{d}\right)$ and sequence of positive reals $h(M)$ such that $\lim _{M \rightarrow \infty} h(M)=$ 0 and for $M \geq 10$

$$
\lim \sup _{N \rightarrow \infty}\left(N^{-d} \sum_{x \in \mathbb{Z}^{d} \cap[0, N(d}\left|v(x, \eta)-\nabla G^{N}(x, \eta, M)\right|^{2}\right)^{\frac{1}{2}} \leq h(M)
$$

Let us now prove lemma 5.2 Let $M \in \mathbb{N}, M \geq 10, M \leq 10^{3} N$. Since $v \in F_{\text {pot }}^{2}$, there exists $u^{M} \in L^{2}(\mu)$ such that

$$
\sum_{i=1}^{d}\left\langle\left|v_{i}-D_{i} u^{M}\right|^{2}\right\rangle \leq 1 / M^{2}
$$

Observe that by the ergodic theorem 3.6 $\eta$-a.s.

$$
\lim \sup _{N \rightarrow \infty}\left(N^{-d} \sum_{x \in \mathbb{Z}^{d} \cap[0, N(d}\left|v(x, \eta)-\nabla u^{M}(x, \eta)\right|^{2}\right)^{\frac{1}{2}} \leq 1 / M
$$

Defining $\alpha_{M}(x)$ as in the subsection 5.1.2 our candidate for $G^{N}$ will be the $\mathrm{E}^{2}\left(T_{N}^{d}\right)$ periodic function with value $([N / M]$ being the integer part of $N / M)$

$$
G^{N}(x, \eta, M)=\left(u^{M}(x, \eta)-[N / M]^{-d} \sum_{y \in\left[0, N / M\left(d \cap \mathbb{Z}^{d}\right.\right.} u^{M}(y, \eta)\right) \alpha_{M}(x / N) \quad \text { on } \quad\left[0, N\left({ }^{d} \cap \mathbb{Z}^{d}\right.\right.
$$

From this point the proof of lemma 5.2 is trivially similar to the one given in subsection 5.1 .2

\subsubsection{Proof of equation (21) of lemma 3.3 in the continuous case}

Let $v \in F_{\text {pot }}^{2}$. We will prove in this subsection that for $\mu$-almost all $\eta \in X$

$$
\lim _{N \rightarrow \infty}\left\|\Pi_{N} v(\eta)-\left(\Pi_{N} v(\eta)\right)_{p o t}\right\|_{£^{2}\left(T_{N}^{d}\right)}=0
$$

Observe that to prove the equation (138) it is sufficient to prove the following lemma

Lemma 5.3. For $\mu$-almost all $\eta \in X$, there exists a sequence $\left(G^{N}(x, \eta, M)\right)_{M, N \in \mathbb{N}}$ of functions in $H^{1}\left(T_{1}^{d}\right)$ and sequence of positive reals $h(M)$ such that $\lim _{M \rightarrow \infty} h(M)=$ 0 and for $M \geq 10$

$$
\lim \sup _{N \rightarrow \infty}\left\|v(N x, \eta)-\nabla G^{N}(x, \eta, M)\right\|_{L^{2}\left([0,1]^{d}\right)} \leq h(M)
$$


Let us now prove lemma 5.3. Since $v \in F_{\text {pot }}^{2}$, for almost all $\eta, v$ admits the following representation $v(x, \eta)=\nabla_{x} u(x, \eta)$, where $u(x, \eta)$ is an element of $H_{l o c}^{1}\left(\mathbb{R}^{d}\right)$ (see subsection [2.2).

Let $M \in \mathbb{N}, M \geq 10$. Let $z \rightarrow g(z)$ be a smooth increasing function on $\mathbb{R}$ such that $g=1$ for $z \geq 1$ and $g=0$ for $z \leq 1 / 2$ and let for $x \in[0,1]^{d}$ $\alpha_{M}(x)=g\left(M \operatorname{dist}\left(x,\left([0,1]^{d}\right)^{c}\right)\right)$. Our candidate for $G^{N}$ will be the $H^{1}\left(T_{1}^{d}\right)$ periodic function with value

$G^{N}(x, \eta, M)=N^{-1}\left(u(N x, \eta)-M^{d} \int_{[0,1 / M]^{d}} u(N y, \eta) d y\right) \alpha_{M}(x) \quad$ on $\quad[0,1]^{d}$

Observe that since $\alpha_{M}(x)$ is null on an open neighborhood of $\mathbb{R}^{d}$ containing the boundary of $[0,1]^{d}, G^{N}$ can be defined as an element of $H^{1}\left(T_{1}^{d}\right)$. Let us write

$$
J_{1}(N, M, \eta)=\left\|\nabla u(N x, \eta)-\nabla G^{N}(x, \eta, M)\right\|_{L^{2}\left([0,1]^{d}\right)}
$$

Observe that

$\nabla G^{N}(x, \eta, M)=\nabla u(N x, \eta) \alpha_{M}(x)+N^{-1}\left(u(N x, \eta)-M^{d} \int_{[0,1 / M]^{d}} u(N y, \eta) d y\right) \nabla \alpha_{M}(x)$

Thus

$$
J_{1}(N, M, \eta) \leq J_{2}(N, M, \eta)+J_{3}(N, M, \eta)
$$

with

$$
J_{2}(N, M, \eta)=\left\|\nabla u(N x, \eta)\left(1-\alpha_{M}(x)\right)\right\|_{L^{2}\left([0,1]^{d}\right)}
$$

and

$$
J_{3}(N, M, \eta)=N^{-1}\left\|\left(u(N x, \eta)-M^{d} \int_{[0,1 / M]^{d}} u(N y, \eta) d y\right) \nabla \alpha_{M}(x)\right\|_{L^{2}\left([0,1]^{d}\right)}
$$

Write

$$
A_{M}=\left\{x \in[0,1]^{d}: \min _{j \in\{1, \ldots d\}} \min \left(x_{j}, 1-x_{j}\right) \leq 1 / M\right\}
$$

Observe that

$$
\begin{aligned}
J_{2}(N, M, \eta) & \leq\|\nabla u(N x, \eta)\|_{L^{2}\left(A_{M}\right)} \\
& \leq C_{d} M^{-\frac{1}{2}}\left(\operatorname{Vol}\left(A_{M}\right)\right)^{-1 / 2}\|\nabla u(N x, \eta)\|_{L^{2}\left(A_{M}\right)}
\end{aligned}
$$

and by the ergodic theorem 3.5] $\eta$-a.s. $\left(\operatorname{Vol}\left(A_{M}\right)\right)^{-1 / 2}\|\nabla u(N x, \eta)\|_{L^{2}\left(A_{M}\right)} \rightarrow$ $\left\langle v^{2}\right\rangle^{\frac{1}{2}}$ as $N \rightarrow \infty$. Thus $\eta$-a.s.

$$
\lim \sup _{N \rightarrow \infty} J_{2}(N, M, \eta) \leq C_{d} M^{-\frac{1}{2}}\left\langle v^{2}\right\rangle^{\frac{1}{2}}
$$

Now let us prove that $\eta-a . s$.

$$
\lim _{M \rightarrow \infty} \lim \sup _{N \rightarrow \infty} J_{3}(N, M, \eta)=0
$$


Since $\left|\nabla \alpha_{M}\right| \leq C_{d} M$ one has

$$
J_{3}(N, M, \eta) \leq C_{d^{\prime}} N^{-1}\left\|M\left(u(N x, \eta)-M^{d} \int_{[0,1 / M]^{d}} u(N y, \eta) d y\right)\right\|_{L^{2}\left(A_{M}\right)}
$$

Let $I(M)=\left\{\left(i_{1}, \ldots, i_{d}\right) \in\{1, \ldots, M\}^{d} ; \min _{j} \min \left(i_{j}-1, M-i_{j}\right)=0\right\}$ and $\left\{B_{i}\right\}_{i \in I(M)}$ the set of cubes covering $A_{M}$ (the $1 / M$-neighborhood of the border of $\left.[0,1]^{d}\right)$. More precisely for $i \in I(M)$,

$$
B_{i}=\left\{x \in[0,1]^{d}: \max _{j}\left|x_{j}-\left(i_{j}-0.5\right) / M\right| \leq 1 /(2 M)\right\}
$$

By the equation (149) one has

$$
J_{3}(N, M, \eta)^{2} \leq C_{d} M^{2} \sum_{i \in I(M)} K_{i}
$$

with

$$
K_{i}=N^{-2} \int_{B_{i}}\left(u(N x, \eta)-M^{d} \int_{[0,1 / M]^{d}} u(N y, \eta) d y\right)^{2} d x
$$

Now using the inequality $(X+Y)^{2} \leq 2 X^{2}+2 Y^{2}$ observe that

$$
\begin{aligned}
K_{i} \leq & 2 N^{-2} \int_{B_{i}}\left(u(N x, \eta)-\left(\operatorname{Vol}\left(B_{i}\right)\right)^{-1} \int_{B_{i}} u(N y, \eta) d y\right)^{2} d x \\
& +2 N^{-2}\left(\operatorname{Vol}\left(B_{i}\right)\right)^{-1}\left(\int_{[0,1 / M]^{d}} u(N y, \eta) d y-\int_{B_{i}} u(N y, \eta) d y\right)^{2}
\end{aligned}
$$

By the Poincaré inequality one has

$$
N^{-2} \int_{B_{i}}\left(u(N x, \eta)-\left(\operatorname{Vol}\left(B_{i}\right)\right)^{-1} \int_{B_{i}} u(N y, \eta) d y\right)^{2} d x \leq C_{d} M^{-2} \int_{B_{i}}(v(N x, \eta))^{2} d x
$$

Thus

$$
\begin{aligned}
J_{3}(N, M, \eta)^{2} \leq & C_{d} M^{-1}\left(\operatorname{Vol}\left(A_{M}\right)\right)^{-1} \int_{A_{M}}(v(N x, \eta))^{2} d x \\
& +C_{d} M^{2} \sum_{i \in I(M)}\left(\operatorname{Vol}\left(B_{i}\right)\right)^{-1} N^{-2}\left(\int_{[0,1 / M]^{d}} u(N y, \eta) d y-\int_{B_{i}} u(N y, \eta) d y\right)^{2}
\end{aligned}
$$

It shall be proven in the paragraph 5.1.4.1 that by the ergodic theorem $\eta-a . s$. for all $i \in I(M)$

$$
\lim _{N \rightarrow \infty} N^{-1}\left|\int_{[0,1 / M]^{d}} u(N y, \eta) d y-\int_{B_{i}} u(N y, \eta) d y\right|=0
$$


It follows that $\eta-a . s$.

$$
\lim \sup _{N \rightarrow \infty} J_{3}(N, M, \eta)^{2} \leq C_{d} M^{-1}<v^{2}>
$$

And taking the limit $M \rightarrow \infty$ one obtains the equation (148). Now combining equations (141), (142), (147) one obtains lemma 5.3 with $h(M)=$ $C_{d} M^{-1 / 2}<v^{2}>^{1 / 2}$.

5.1.4.1 Proof of equation (156) Let $a, b \in I(M) \times I(M), q \in \mathbb{N}^{*}$ and $w \in\{1, \ldots, d\}$ such that $b=a+q e_{w}$. Observing that

$$
u\left(N\left(x+q e_{w} / M\right), \eta\right)-u(N x, \eta)=\int_{t \in[0,1]} v\left(N\left(x+q t e_{w} / M\right), \eta\right) \cdot\left(q e_{w} / M\right) d t
$$

we obtain

$$
\begin{aligned}
N^{-1} \int_{B_{b}} u(N x, \eta) d x= & N^{-1} \int_{B_{a}} u(N x, \eta) d x \\
& +\int_{t \in[0,1]} \int_{B_{a}} v\left(N\left(x+q t e_{w} / M\right), \eta\right) \cdot\left(q e_{w} / M\right) d x d t
\end{aligned}
$$

Now let us write $\partial^{w} B_{a}$ the lower face of the cube $B_{a}$ orthogonal to $e_{w}$ :

$$
\partial^{w} B_{a}:=\left\{x \in B_{a}: x . e_{w}=\left(a_{w}-0.5\right) / M-1 /(2 M)\right\}
$$

Now we decompose $x \in B_{a}$ as $x=x^{w}+y_{w} e_{w} / M$ with $x^{w} \in \partial^{w} B_{a}$ and $y_{w} \in[0,1]$, using this change of variable we obtain

$$
\begin{aligned}
\int_{t \in[0,1]} & \int_{B_{a}} v\left(N\left(x+q t e_{w} / M\right), \eta\right) \cdot\left(q e_{w} / M\right) d x d t \\
& =\int_{t \in[0,1]} \int_{y_{w} \in[0,1], x^{w} \in \partial^{w} B_{a}} v\left(N\left(x^{w}+\left(t q+y_{w}\right) e_{w} / M\right), \eta\right) \cdot\left(q e_{w} / M^{2}\right) d x^{w} d t d y_{w}
\end{aligned}
$$

Now using the change of variable $s=\left(t q+y_{w}\right) / M$ we obtain from (159) that

$$
\begin{aligned}
\int_{t \in[0,1]} & \int_{B_{a}} v\left(N\left(x+q t e_{w} / M\right), \eta\right) \cdot\left(q e_{w} / M\right) d x d t \\
& =\int_{y_{w} \in[0,1]} \int_{s \in\left[y_{w} / M,\left(q+y_{w}\right) / M\right]} \int_{x^{w} \in \partial^{w} B_{a}} v\left(N\left(x^{w}+s e_{w}\right), \eta\right) \cdot\left(e_{w} / M\right) d x^{w} d s d y_{w} \\
& =\int_{s \in[1 / M, q / M]} \int_{x^{w} \in \partial^{w} B_{a}} v\left(N\left(x^{w}+s e_{w}\right), \eta\right) \cdot\left(e_{w} / M\right) d x^{w} d s \\
& +\int_{s \in[0,1 / M]} \int_{x^{w} \in \partial^{w} B_{a}} v\left(N\left(x^{w}+s e_{w}\right), \eta\right) \cdot e_{w} s d x^{w} d s \\
& +\int_{s \in[q / M,(q+1) / M]} \int_{x^{w} \in \partial^{w} B_{a}} v\left(N\left(x^{w}+s e_{w}\right), \eta\right) \cdot e_{w}(1+q-M s) d x^{w} d s
\end{aligned}
$$


Thus using the decomposition $[1 / M, q / M]=\cup_{k=1}^{q-1}[k / M,(k+1) / M]$, it follows from (160) and (158) by obvious change of variables that

$$
\begin{aligned}
N^{-1} \int_{B_{b}} u(N x, \eta) d x= & N^{-1} \int_{B_{a}} u(N x, \eta) d x+M^{-1} \sum_{k=1}^{q-1} \int_{B_{a}+k e_{w}} v(N x, \eta) \cdot e_{w} d x \\
& +\int_{B_{a}}\left(x \cdot e_{w}-a \cdot e_{w} / M+1 / M\right) v(N x, \eta) \cdot e_{w} d x \\
& +\int_{B_{b}}\left(-x \cdot e_{w}+b \cdot e_{w} / M\right) v(N x, \eta) \cdot e_{w} d x
\end{aligned}
$$

Since $\langle v\rangle=0$ one has $\eta$-a.s.

$$
\lim _{N \rightarrow \infty} \sum_{k=1}^{q-1} \int_{B_{a}+k e_{w}} v(N x, \eta) \cdot e_{w} d x=0
$$

Now for $P \in \mathbb{N}^{*}\left(P \geq M^{2}\right)$ write $E_{k}=\left\{x \in B_{b}:(k-1) / P<-x . e_{w}+\right.$ b. $\left.e_{w} / M \leq k / P\right\}$. Note that $\left(E_{k}\right)_{1 \leq k \leq P}$ is a partition of $B_{b}$ (observe that by equation (150) $b / M$ is not the center of $B_{b}$ but the upper edge of the cube). Thus we obtain

$$
\begin{aligned}
\int_{B_{b}}\left(-x \cdot e_{w}+b \cdot e_{w} / M\right) v(N x, \eta) \cdot e_{w} d x \leq & \sum_{k=1}^{P} \int_{E_{k}}(k / P) v(N x, \eta) \cdot e_{w} d x \\
& +1 / P \int_{B_{b}}\left|v(N x, \eta) \cdot e_{w}\right| d x
\end{aligned}
$$

It follows by the ergodic theorem that $\eta$-a.s.

$$
\lim \sup _{N \rightarrow \infty}\left|\int_{B_{b}}\left(-x \cdot e_{w}+b \cdot e_{w} / M\right) v(N x, \eta) \cdot e_{w} d x\right| \leq P^{-1} M^{-d}<\left|v \cdot e_{w}\right|>
$$

And taking the limit $P \rightarrow \infty$ one obtains that

$$
\lim \sup _{N \rightarrow \infty}\left|\int_{B_{b}}\left(-x \cdot e_{w}+b \cdot e_{w} / M\right) v(N x, \eta) \cdot e_{w} d x\right|=0
$$

Similarly one obtains that

$$
\lim \sup _{N \rightarrow \infty}\left|\int_{B_{a}}\left(x \cdot e_{w}-a \cdot e_{w} / M+1 / M\right) v(N x, \eta) \cdot e_{w} d x\right|=0
$$

From (161), (162), (165) and (166) one deduces that for $a, b \in I(M) \times I(M)$, $q \in \mathbb{N}^{*}$ and $w \in\{1, \ldots, d\}$ such that $b=a+q e_{w}$. one has

$$
\lim \sup _{N \rightarrow \infty}\left|N^{-1} \int_{B_{b}} u(N x, \eta) d x-N^{-1} \int_{B_{a}} u(N x, \eta) d x\right|=0
$$

And since any two distinct points of $I(M)$ can be connected by a finite number of steps of such translations one obtains the equation (156) 


\subsubsection{Proof of equation (22) of lemma 3.3 in the continuous case}

Let $p \in F_{\text {sol }}^{2}$. We will prove in this subsection that for $\mu$-almost all $\eta \in X$

$$
\lim _{N \rightarrow \infty}\left\|\Pi_{N} p(\eta)-\left(\Pi_{N} p(\eta)\right)_{s o l}\right\|_{\complement^{2}\left(T_{N}^{d}\right)}=0
$$

Observe that to prove the equation (168), it is sufficient to prove the following lemma

Lemma 5.4. For $\mu$-almost all $\eta \in X$, there exists a sequence $\left(K^{N}(x, \eta, M)\right)_{M, N \in \mathbb{N}}$ of skew symmetric matrices with coefficients in $H^{1}\left(T_{1}^{d}\right)$ and a sequence of positive reals $h(M)$ such that $\lim _{M \rightarrow \infty} h(M)=0$ and for $M \geq 10$

$$
\lim \sup _{N \rightarrow \infty}\left\|p(N x, \eta)-\operatorname{div} K^{N}(x, \eta, M)\right\|_{L^{2}\left([0,1]^{d}\right)} \leq h(M)
$$

Since $p \in F_{\text {sol }}^{2}$, it is easy to prove from Weyl decomposition that there exists a finite sequence $h_{i, j} \in F_{p o t}^{2},(i, j) \in\{1, \ldots d\}^{2}$ such that $h_{i, j}=-h_{j, i}$ and $\eta$-a.s., $(p)_{i}=\sum_{j=1}^{d} h_{i, j} . e_{j}$ ( $F_{\text {pot }}$ is orthogonal to the set of such vectors and any element of $\mathrm{E}^{2}(X, \mu)$ orthogonal to the set of such vectors is in $\left.\mathrm{E}_{\text {pot }}^{2}(X, \mu)\right)$. Write $H_{i, j}$ the scalar potentials associated to $h_{i, j}$, then it follows that $\eta$-a.s., $\operatorname{div}_{x} H_{i, j}(x, \eta)=h_{i, j}(x, \eta)$. Thus $H$ is a $d \times d$ skew symmetric matrix with elements in $H_{l o c}^{1}\left(\mathbb{R}^{d}\right)$ such that $\eta$-a.s., $p(x, \eta)=\operatorname{div} H(x, \eta)$.

For $M \in \mathbb{N}, M \geq 10$, defining $\alpha_{M}(x)$ as in the subsection 5.1.4 our candidate for $K^{N}$ will be the skew symmetric $T_{1}^{d}$-periodic matrix:

$K^{N}(x, \eta, M):=N^{-1}\left(H(N x, \eta)-M^{d} \int_{[0,1 / M]^{d}} H(N y, \eta) d y\right) \alpha_{M}(x) \quad$ on $\quad[0,1]^{d}$

From this point the proof of lemma 5.4 is trivially similar to the one given in subsection 5.1.4.

\subsection{Applications}

In this subsection we will prove theorem 4.4. We will first prove equation (85), the equations (86) and (87) will be implied by the first one.

\subsubsection{Proof of the variational formula (85)}

Let us write $v_{l}^{E} \in F_{p}^{2}$ ot the solution of the equation (70) and (using the linearity of $v_{l}^{E}$ in $l$ one can define $v_{\text {. }}^{E}$ as a matrix by $v^{E} l=v_{l}^{E}$ ). Let us first prove the following lemma

\section{Lemma 5.5.}

$$
\sigma(a,-E, \mu)={ }^{t} \sigma(a, E, \mu)
$$


Proof. We shall adapt the proof given by J. R. Norris [Nor97 for the periodic case. Let $l, k \in \mathbb{R}^{d}$. Since $(a+E)\left(l+v_{l}^{E}\right) \in \mathrm{E}_{\text {sol }}^{2}(X, \mu)$ and $(a-E)\left(k+v_{k}^{-E}\right) \in$ $\mathrm{E}_{\text {sol }}^{2}(X, \mu)$, by the Weyl decomposition (8), there exists $q, h \in F_{\text {sol }}^{2}$ and $t, s \in \mathbb{R}^{d}$ such that

$$
t-q=(a+E)\left(l+v_{l}^{E}\right)
$$

and

$$
s-h=(a-E)\left(k+v_{k}^{-E}\right)
$$

Observe that by integration with respect to the measure $\mu$, one obtains that

$$
t=\sigma(a, E, \mu) l
$$

and

$$
s=\sigma(a,-E, \mu) k
$$

For $f, g \in \mathrm{E}^{2}(X, \mu)$ we write $\langle f, g\rangle=\left\langle{ }^{t} f g\right\rangle$. Then observe that

$$
\begin{aligned}
\langle\sigma(a,-E, \mu) k, l\rangle & =\langle s, l\rangle=\left\langle s-h, l+v_{l}^{E}\right\rangle \\
& =\left\langle(a-E)\left(k+v_{k}^{-E}\right), l+v_{l}^{E}\right\rangle=\left\langle k+v_{k}^{-E},(a+E)\left(l+v_{l}^{E}\right)\right\rangle \\
& =\left\langle k+v_{k}^{-E}, t-q\right\rangle=\langle k, t\rangle \\
& =\langle k, \sigma(a, E, \mu) l\rangle
\end{aligned}
$$

Which proves that ${ }^{t} \sigma(a,-E, \mu)=\sigma(a, E, \mu)$ and henceforth the lemma.

Let $\xi, l \in \mathbb{R}^{d}$, we will now prove that

$$
|\xi-\sigma(a, E, \mu) l|_{\sigma_{\mathrm{sym}}^{-1}(a, E, \mu)}^{2}=\inf _{\psi, p \in F_{p o t} \times F_{\text {sol }}}\left\langle|\xi-p-(a+E)(l-\psi)|_{a^{-1}}^{2}\right\rangle
$$

We will write $\sigma_{\text {sym }}$ is the symmetric part of $\sigma(a, E, \mu)$. Let us define

$$
\left.\psi_{0}:=v_{.}^{E}\left(l+\frac{1}{2} \sigma_{\mathrm{sym}}^{-1}(\xi-\sigma l)\right)\right)-v_{.}^{-E} \frac{1}{2} \sigma_{\mathrm{sym}}^{-1}(\xi-\sigma l)
$$

and

$$
p_{0}:=\xi-(a+E)\left(l-\psi_{0}\right)-a\left(I_{d}-v^{-E}\right) \sigma_{\mathrm{sym}}^{-1}(\xi-\sigma l)
$$

Observe also that since

$$
\xi-p_{0}-(a+E)\left(l-\psi_{0}\right)=a\left(I_{d}-v^{-E}\right) \sigma_{\mathrm{sym}}^{-1}(\xi-\sigma l)
$$

And (using lemma 5.5)

$$
\left\langle{ }^{t}\left(I_{d}-v^{-E}\right) a\left(I_{d}-v^{-E}\right)\right\rangle=\sigma_{\mathrm{sym}}(a,-E, \mu)=\sigma_{\mathrm{sym}}(a, E, \mu)=\sigma_{\mathrm{sym}}
$$


One obtains that

$$
\left\langle\left|\xi-p_{0}-(a+E)\left(l-\psi_{0}\right)\right|_{a^{-1}}^{2}\right\rangle=|\xi-\sigma(a, E, \mu) l|_{\sigma_{\text {sym }}^{-1}(a, E, \mu)}^{2}
$$

Moreover $\psi_{0} \in F_{\text {pot }}^{2}$ and $p_{0} \in F_{\text {sol }}^{2}$ since

$$
\begin{aligned}
p_{0}= & \xi-(a+E) l-a \sigma_{\mathrm{sym}}^{-1}(\xi-\sigma l) \\
& +(a+E)\left(I_{d}+v_{.}^{E}\right)\left(l+\frac{1}{2} \sigma_{\mathrm{sym}}^{-1}(\xi-\sigma l)\right) \\
& +(a-E)\left(I_{d}+v^{-E}\right) \frac{1}{2} \sigma_{\mathrm{sym}}^{-1}(\xi-\sigma l)
\end{aligned}
$$

And by the equation (180),$a^{-1}\left(\xi-p_{0}-(a+E)\left(l-\psi_{0}\right)\right)$ is orthogonal in $\mathrm{E}^{2}(X, \mu)$ to $F_{\text {sol }}^{2}$ and the space $\left\{(a+E) v: v \in F_{\text {pot }}^{2}\right\}$, it follows that the variational formula (177) is valid and the minimum is reached at $p_{0}$ and $\psi_{0}$.

\subsubsection{Proof of the variational formulas (86) and (87)}

One obtains the variational formula (86) from the variational formula (85) by observing that

$$
\inf _{\xi \in \mathbb{R}^{d}, \xi \perp l}|\xi-\sigma(a, E, \mu) l|_{\sigma_{\mathrm{sym}}^{-1}(a, E, \mu)}^{2}={ }^{t} l \sigma_{\mathrm{sym}}(a, E, \mu) l
$$

One obtains the variational formula (87) by taking $l=0$ in (85).

\subsubsection{Proof of lemma 4.7}

Gift $\mathrm{E}^{2}(X, \mu)$ with the scalar product $(f, g)_{H}=\sum_{i=1}^{d}<\xi_{i} f_{i} g_{i}>$ to obtain an Hilbert space. By the variational formula (95), ${ }^{t} l D(\xi, \mu) l$ is the norm of the H-orthogonal projection of $l$ on the subspace of $\mathrm{E}^{2}(X, \mu)$ H-orthogonal to $F_{\text {pot }}^{2}$. It follows that there exists an unique $v_{l} \in F_{\text {pot }}^{2}$ linear in $l$ realizing the minimum of (95) and such that $\left(l+v_{l}\right)$ is H-orthogonal to $F_{\text {pot }}^{2}$.

Thus the vector for $l \in \mathbb{R}^{d}$ the vector field $p_{l}$ defined by

$$
p_{l}=\xi_{i}\left(I_{d}+v_{.}\right)(D(\xi, \mu))^{-1} l-l
$$

verifies $\left\langle p_{l}\right\rangle=0$ and is orthogonal to $F_{p o t}^{2}$, thus by the theorem 2.6 it is an element of $F_{\text {sol }}^{2}$. Moreover observing that

$$
\left\langle\xi_{i}^{-1}\left(l_{i}+\left(p_{l}\right)_{i}\right)^{2}\right\rangle={ }^{t} l D(\xi, \mu)^{-1} l
$$

and since the vector $q$ defined by $q_{i}=\xi_{i}^{-1}\left(l_{i}+\left(p_{l}\right)_{i}\right)=\left(I_{d}+v_{.}\right)(D(\xi, \mu))^{-1} l$ is orthogonal to $F_{\text {sol }}^{2}$ one obtains that the variational formula (4.7) is true and that its minimum is reached at $p_{l}$. 
Acknowledgments Part of this work was supported by the Aly Kaufman fellowship. The author would like to thank Dmitry Ioffe for his hospitality during his stay at the Technion, for suggesting this problem and for stimulating and helpful discussions. Thanks are also due to the referee for many useful comments.

\section{References}

[BLP78] A. Bensoussan, J. L. Lions, and G. Papanicolaou. Asymptotic analysis for periodic structure. North Holland, Amsterdam, 1978.

[CI01] Pietro Caputo and Dmitry Ioffe. Finite volume approxiamtion of the effective diffusion matrix: the case of independent bond disorder. preprint arXiv:math.PR/0110215, 2001.

[DGI00] J-D. Deuschel, G. Giacomin, and D. Ioffe. Large deviations and concentration properties for $\nabla \phi$ interface models. Probab. Theory Related Fields, 117:49-111, 2000.

[DS67] N. Dunford and J. T. Schwartz. Linear Operators, Part I. Interscience plublishers, 1967.

[FP94] A. Fannjiang and G.C. Papanicolaou. Convection enhanced diffusion for periodic flows. SIAM J. Appl. Math., 54:333-408, 1994.

[FP96] A. Fannjiang and G.C. Papanicolaou. Diffusion in turbulence. Probab. Theory Related Fields, 105(3):279-334, 1996.

[GOS01] G.B. Giacomin, S. Olla, and H. Spohn. Equilibrium fluctuation for a Ginzburg-Landau $\nabla \phi$ interface model. Annals of Probability, 2001.

[JKO91] V. V. Jikov, S. M. Kozlov, and O. A. Oleinik. Homogenization of Differential Operators and Integral Functionals. SpringerVerlag, 1991.

[Koz80] S.M. Kozlov. Averaging of random operators. Math USSR Sbornik, 37:167-180, 1980.

[Koz85] S.M. Kozlov. The method of averaging and walks in inhomogeneous environments. Russian Math. Surveys, 2(40):73-145, 1985.

[KV86] C. Kipnis and S.R.S. Varadhan. Central limit theorem for additive functional of reversible markov processes and application to simple exclusion. Comm. Math. Phys., 104:1-19, 1986. 
[LOV01] C. Landim, S. Olla, and S.R.S. Varadhan. Finite-dimensional approximation of the self-diffusion coefficient. Comm. Math. Phys., 224:302-321, 2001.

[MFGW89] A. De Masi, P. A. Ferrari, S. Goldstein, and W.D. Wick. An invariance principle for reversible markov processes. application to random motions in random environments. Journal of Statistical Physics, 55(3/4):787-855, 1989.

[Nor97] J.R. Norris. Long-time behaviour of heat flow: Global estimates and exact asymptotics. Arch. Rational Mech. Anal., 140:161195, 1997.

[Oll94] S. Olla. Homogenization of Diffusion Processes in Random Fields. Ecole Polytechnique, 1994. Cours Ecole Polytechnique.

[OS95] H. Osada and T. Satoh. An invariance principle for nonsymmetric markov processes and reflecting diffusions in random domains. Probab. Theory Relat. Fields, 101:45-63, 1995.

[Pia02] Andrey Piatnitski. Private discussion. 2002.

[PV79] G. Papanicolaou and S.R.S. Varadhan. Boundary value problems with rapidly oscillating random coefficients. In Colloquia Mathematica Societatis János Bolay, volume 27, 1979. 